\title{
Total Syntheses of Didehydrostemofoline (Asparagamine A) and Isodidehydrostemofoline
}

\author{
Markus Brüggemann, Andrew I. McDonald, ${ }^{1 \mathrm{a}}$ Larry E. Overman, ${ }^{*}$ Mark D. Rosen, ${ }^{1 \mathrm{~b}}$ \\ Lothar Schwink $^{1 \mathrm{c}}$ and Jeremy P. Scott ${ }^{1 \mathrm{~d}}$ \\ Department of Chemistry, 516 Rowland Hall, University of California, Irvine, CA 92697-2025
}

\section{Supporting Information}

\section{Experimental procedures for key steps; copies of ${ }^{1} \mathrm{H}$ and ${ }^{13} \mathrm{C}$ NMR spectra for all new compounds (34 pages)}

General. All reactions were carried out under argon or nitrogen in flame-dried glassware. THF, $\mathrm{Et}_{2} \mathrm{O}, \mathrm{DME}, \mathrm{CH}_{2} \mathrm{Cl}_{2}$, toluene, $\mathrm{MeOH}, \mathrm{Et}_{3} \mathrm{~N}, \mathrm{MeCN}$, pyridine and $i \mathrm{Pr}_{2} \mathrm{NH}$ (for LDA) were taken from a solvent purification system ${ }^{2}$ from GlassContour. ${ }^{3}$ IBX and DMP were prepared according to the usual procedures. ${ }^{4} \mathrm{CeCl}_{3}$ was dried from the heptahydrate, ${ }^{5}$ vinylmagnesium bromide was prepared from vinylbromide and magnesium. ${ }^{6}$ All other chemical were purchased at the highest quality available and used as received. Flash column chromatography was performed on EM Science silica gel 60, 0.040-0.063 mm in the typical manner; ${ }^{7}$ for compounds containing a basic nitrogen functionality, deactivated silica gel that had been open to the air overnight was used. The NMR spectra were recorded on Bruker DRX 400 and DRX 500, IR spectra were recorded on Perkin-Elmer Series 1600 and ASI React-IR 1000, and high resolution mass spectra were obtained from Waters Instruments Autospec E and LCT. Microanalyses were performed at Atlantic Micro Labs, Atlanta, GA.

Pyrrole 9. According to the procedure of Bellamy, ${ }^{8}$ a solution of 4-methoxypyridine $N$ oxide hydrate $(12.0 \mathrm{~g}, 83.9 \mathrm{mmol}), \mathrm{CuSO}_{4} \cdot 5 \mathrm{H}_{2} \mathrm{O}(240 \mathrm{~g}, 961 \mathrm{mmol})$ and deionized $\mathrm{H}_{2} \mathrm{O}(3.5 \mathrm{~L})$ in a beaker was irradiated with a $450 \mathrm{~W}$ medium pressure $\mathrm{Hg}$ lamp for $24 \mathrm{~h}$ at room temperature. The lamp was placed in a jacketed quartz sleeve which was submerged in the reaction solution and cooled with recirculating $20{ }^{\circ} \mathrm{C}_{2} \mathrm{O}$. The reaction solution was then saturated with $\mathrm{NaCl}$ and extracted with $\mathrm{CHCl}_{3}(3 \square 0.50 \mathrm{~L})$. The combined organic extracts were washed with brine $(0.50$ $\mathrm{L})$, dried $\left(\mathrm{MgSO}_{4}\right)$ and filtered through a plug of silica gel. The filtrate was discarded and the plug of silica gel was flushed with $10: 1 \mathrm{CHCl}_{3}-\mathrm{MeOH}$ and the filtrate was concentrated in vacuo

\footnotetext{
${ }^{1}$ Current addresses: (a) Cytokinetics, 280 East Grand Avenue, South San Franicsco, CA 94080; (b) Johnson and Johnson, Pharmaceutical Research and Development, 3210 Merryfield Row, San Diego, CA 92121; (c) Aventis, Haan, Germany; (d) Merck, Sharp \& Dohme Limited, Hertford Road, Hoddeson EN11 9BU England

${ }^{2}$ Pangborn, A. B.; Giardello, M. A.; Grubbs, R. H.; Rosen, R. K.; Timmers, F. J. Organometallics 1996, $15,1518$.

${ }^{3}$ For details see: http://www.glasscontour.com.

${ }^{4}$ a) Frigerio, M.; Santagostino, M.; Sputore, S. J. Org. Chem. 1999, 64, 4537. b) Ireland, R. E., Liu, L. J. Org. Chem. 1993, 58, 2899.

${ }^{5}$ Encyclopedia of Reagents for Organic Synthesis; Parquette, L. A., Ed.; John Wiley \& Sons, Chichester, 1995, vol. $2,1031$.

${ }^{6}$ Normat, H. Bull. Soc. Chim. Fr. (2), 1957, 728.

${ }^{7}$ Still, W. C.; Kahn, M.; Mitra, A. J. Org. Chem. 1978, 43, 2923.

${ }^{8}$ Bellamy, F.; Martz, P.; Streith, J. Heterocycles 1975, 3, 395.
} 
to provide a brown residue $(4.00 \mathrm{~g}, 32.0 \mathrm{mmol}, 38 \%)$ which was used directly in the following step.

A solution of this crude product, $\mathrm{BOC}_{2} \mathrm{O}(11.0 \mathrm{~g}, 50.4 \mathrm{mmol}), 4-\mathrm{N}, \mathrm{N}-$ dimethylaminopyridine $(50 \mathrm{mg}, 0.41 \mathrm{mmol})$ and $\mathrm{CH}_{2} \mathrm{Cl}_{2}(50 \mathrm{~mL})$ was maintained at room temperature for $1.5 \mathrm{~h}$. The solution was concentrated in vacuo, the resulting crude oil was diluted with EtOAc $(0.30 \mathrm{~L})$, and the resulting solution was washed with aqueous $\mathrm{HCl}(0.5 \mathrm{~N}, 0.20 \mathrm{~L})$ and brine $(0.10 \mathrm{~L})$, dried $\left(\mathrm{MgSO}_{4}\right)$, filtered and the filtrate was concentrated in vacuo. Chromatography of this residue (50:50 EtOAc-hexanes) on silica gel provided $6.50 \mathrm{~g}$ (28.8 mmol, 34\% over 2 steps) of 1-(tert-butoxycarbonyl)-3-methoxypyrrole-2-carboxyaldehyde as a colorless solid: $\mathrm{mp} 83-85^{\circ} \mathrm{C}$; IR (neat) 2990, 2900, 1740, 1650, 1555, 1440, 1160, 1100, 970 $\mathrm{cm}^{-1}$; ${ }^{1} \mathrm{H} \mathrm{NMR}\left(\mathrm{CDCl}_{3}, 400 \mathrm{MHz}\right) \square 10.21(\mathrm{~s}, 1 \mathrm{H}), 7.35(\mathrm{~d}, J=3.7 \mathrm{~Hz}, 1 \mathrm{H}), 6.08$ (d, $J=3.7 \mathrm{~Hz}$, $1 \mathrm{H}), 3.91$ (s, $3 \mathrm{H}), 1.59$ (s, $9 \mathrm{H}) \mathrm{ppm} ;{ }^{13} \mathrm{C} \mathrm{NMR}\left(\mathrm{CDCl}_{3}, 100 \mathrm{MHz}\right) \square 180.7,158.4,148.3,126.6$, 118.5, 99.2, 85.6, 58.4, 27.9 ppm. Anal. Calcd for $\mathrm{C}_{11} \mathrm{H}_{15} \mathrm{NO}_{4}$ : C, 58.66; $\mathrm{H}, 6.71 ; \mathrm{N}, 6.22$. Found: C, 58.75; H, 6.66; N, 6.34 .

Sodium borohydride $(110 \mathrm{mg}, 2.91 \mathrm{mmol}$ ) was added slowly over $5 \mathrm{~min}$ to a solution of this pyrrole $(450 \mathrm{mg}, 2.00 \mathrm{mmol})$ and $\mathrm{MeOH}(4.0 \mathrm{~mL})$ at $0{ }^{\circ} \mathrm{C}$. After $30 \mathrm{~min}$, the reaction was quenched by pouring into $\mathrm{Et}_{2} \mathrm{O}(50 \mathrm{~mL})$ and saturated aqueous $\mathrm{NaHCO}_{3}(20 \mathrm{~mL})$. The layers were separated and the organic layer was washed with brine $(20 \mathrm{~mL})$, dried $\left(\mathrm{MgSO}_{4}\right)$, filtered and the filtrate was concentrated in vасио. Residual 9, a colorless oil, was used without further purification.

Cycloadducts 10 and 11. Crude pyrrole alcohol 9 and ethyl $(E)-3$-nitroacrylate ${ }^{9}$ (350 $\mathrm{mg}, 2.41 \mathrm{mmol})$ were dissolved in EtOAc $(5.0 \mathrm{~mL})$ and then concentrated in vacuo. The resulting viscous oil was maintained at room temperature for $5 \mathrm{~h}$. The crude cycloadducts were then rapidly dissolved in EtOAc $(2.0 \mathrm{~mL})$ and added to a mixture of $10 \% \mathrm{Pd}$ on activated carbon $(200$ $\mathrm{mg})$ and EtOAc $(7.0 \mathrm{~mL})$ that was presaturated with $\mathrm{H}_{2}$ at 40 psi. The resulting mixture was repressurized (40 $\mathrm{psi}_{2}$ ) and maintained at room temperature for $1.25 \mathrm{~h}$. The reaction mixture was depressurized and filtered though a pad of silica gel (EtOAc rinse) and the eluent was concentrated in vacuo. The crude oil was chromatographed (83:17 80:20 hexanes-EtOAc) on silica gel to provide $550 \mathrm{mg}$ (1.47 mmol, 73\%, over 3 steps) of 10 and $100 \mathrm{mg}$ (267 $\square \mathrm{mol}, 13 \%$, over 3 steps) of 11 as slightly yellow solids. 10: $\mathrm{mp} 94-96^{\circ} \mathrm{C}$; IR (film) 3428, 2981, 2936, 2834, 1732, 1682, 1556, $1454 \mathrm{~cm}^{-1}$; ${ }^{1} \mathrm{H}$ NMR $\left(\mathrm{CDCl}_{3}, 500 \mathrm{MHz}\right) \square 5.28(\mathrm{~d}, J=4.1 \mathrm{~Hz}, 1 \mathrm{H})$, $4.83(\mathrm{~d}, J=6.0 \mathrm{~Hz}, 1 \mathrm{H}), 4.45(\mathrm{br} \mathrm{s}, 1 \mathrm{H}), 4.35(\mathrm{~d}, J=13.2 \mathrm{~Hz}, 1 \mathrm{H}), 4.28(\mathrm{~d}, J=13.2 \mathrm{~Hz}, 1 \mathrm{H})$, $4.25-4.13(\mathrm{~m}, 2 \mathrm{H}), 4.06(\mathrm{~d}, J=3.9 \mathrm{~Hz}, 1 \mathrm{H}), 3.83(\mathrm{dd}, J=9.7,3.4 \mathrm{~Hz}, 1 \mathrm{H}), 3.28(\mathrm{~s}, 3 \mathrm{H})$, 2.38-2.28 (m, $1 \mathrm{H}), 1.46-1.39(\mathrm{~m}, 1 \mathrm{H}), 1.42(\mathrm{~s}, 9 \mathrm{H}), 1.30(\mathrm{t}, J=7.1 \mathrm{~Hz}, 3 \mathrm{H}) \mathrm{ppm} ;{ }^{13} \mathrm{C}$ NMR $\left(\mathrm{CDCl}_{3}, 125 \mathrm{MHz}\right) \square 168.3,153.3,88.7,82.4,79.3,76.7,74.5,62.2,61.4,59.4,49.4,32.9,27.8$, 13.9 ppm; HRMS (FAB) $m / z 375.1776$ (calcd for $\mathrm{C}_{16} \mathrm{H}_{26} \mathrm{~N}_{2} \mathrm{O}_{8}+\mathrm{H}^{+}$, 375.1767). Anal. Calcd for $\mathrm{C}_{16} \mathrm{H}_{26} \mathrm{~N}_{2} \mathrm{O}_{8}$ : C, 51.33; H, 7.00; N, 7.48. Found: C, 51.52; H, 7.04; N, 7.48. 11: mp 89-91 ${ }^{\circ} \mathrm{C}$; IR (film) 3424, 2982, 2937, 2835, 1704, 1564, $1455 \mathrm{~cm}^{-1} ;{ }^{1} \mathrm{H} \mathrm{NMR}\left(\mathrm{CDCl}_{3}, 500 \mathrm{MHz}\right) \square 5.40(\mathrm{~d}, J$ $=4.8 \mathrm{~Hz}, 1 \mathrm{H}), 4.59(\mathrm{~d}, J=5.5 \mathrm{~Hz}, 1 \mathrm{H}), 4.47-4.44(\mathrm{~m}, 1 \mathrm{H}), 4.36-4.31(\mathrm{~m}, 1 \mathrm{H}), 4.26-4.19(\mathrm{~m}$, $3 \mathrm{H}), 3.93(\mathrm{dd}, J=9.8,3.0 \mathrm{~Hz}, 1 \mathrm{H}), 3.75(\mathrm{~d}, J=4.8 \mathrm{~Hz}, 1 \mathrm{H}), 3.26(\mathrm{~s}, 3 \mathrm{H}), 2.38-2.31(\mathrm{~m}, 1 \mathrm{H})$, $1.58(\mathrm{dd}, J=13.0,3.2 \mathrm{~Hz}, 1 \mathrm{H}), 1.41(\mathrm{~s}, 9 \mathrm{H}), 1.31(\mathrm{t}, J=7.2 \mathrm{~Hz}, 3 \mathrm{H}) \mathrm{ppm} ;{ }^{13} \mathrm{C} \mathrm{NMR}\left(\mathrm{CDCl}_{3}\right.$, $125 \mathrm{MHz}) \square 170.8,153.6,83.5,82.6,80.9,74.4,62.4,60.9,59.7,59.0,51.1,36.1,28.4,14.6$

\footnotetext{
${ }^{9}$ McMurry, J. E.; Musser, J. H. Org. Synth. 1977, 56, 65.
} 
ppm; HRMS (FAB) $\mathrm{m} / z 375.1779$ (calcd for $\mathrm{C}_{16} \mathrm{H}_{27} \mathrm{~N}_{2} \mathrm{O}_{8}+\mathrm{H}^{+}, 375.1767$ ). Anal. Calcd for $\mathrm{C}_{16} \mathrm{H}_{26} \mathrm{~N}_{2} \mathrm{O}_{8}$ : C, 51.33; H, 7.00; N, 7.48. Found: C, 51.50; H, 7.01; N, 7.46.

aza-Cope-Mannich precursor 14 and product ketone 15. Rigorously dried $\mathrm{CeCl}_{3}$ $(6.55 \mathrm{~g}, 26.6 \mathrm{mmol})$ and THF $(0.15 \mathrm{~L})$ were stirred at room temperature for $18 \mathrm{~h}$. A solution of freshly prepared vinylmagnesium bromide and THF $(1.0 \mathrm{M}, 27 \mathrm{~mL}, 27 \mathrm{mmol})$ was then added to the slurry at $-78{ }^{\circ} \mathrm{C}$. After $30 \mathrm{~min}$, a solution of $\mathbf{1 3}(3.87 \mathrm{~g}, 9.06 \mathrm{mmol})$ and THF $(9.0 \mathrm{~mL})$ was added by cannula to the reaction mixture. After $15 \mathrm{~min}$, the reaction was quenched by adding aqueous $\mathrm{HCl}(1 \mathrm{~N}, 20 \mathrm{~mL})$. The layers were separated and the organic layer was washed with brine $(30 \mathrm{~mL})$, dried $\left(\mathrm{MgSO}_{4}\right)$, filtered and the filtrate was concentrated in vacuo. The resulting oil was chromatographed (95:5 $\square$ 91:9 hexanes-EtOAc) on silica gel to provide $3.50 \mathrm{~g}$ (7.68 mmol, 85\%) of the $N$-Boc derivative of 14 as a colorless oil: IR (film) 3460, 2942, 2865, 1710, $1695,1455,1365,1125 \mathrm{~cm}^{-1} ;{ }^{1} \mathrm{H} \mathrm{NMR}\left(\mathrm{CDCl}_{3}, 500 \mathrm{MHz}\right) \square 5.75(\mathrm{ddd}, J=16.9,10.6,1.1 \mathrm{~Hz}, 1$ H), $5.46(\mathrm{~s}, 1 \mathrm{H}), 5.34(\mathrm{dd}, J=16.9,1.9 \mathrm{~Hz}, 1 \mathrm{H}), 5.04(\mathrm{dd}, J=10.6,1.9 \mathrm{~Hz}, 1 \mathrm{H}), 4.62(\mathrm{~d}, J=$ $10.5 \mathrm{~Hz}, 1 \mathrm{H}), 4.43$ (dd, $J=9.9,3.7 \mathrm{~Hz}, 1 \mathrm{H}), 4.32$ (t, $J=5.5 \mathrm{~Hz}, 1 \mathrm{H}), 4.00$ (d, $J=11.2 \mathrm{~Hz}, 1$ $\mathrm{H}), 3.39(\mathrm{~s}, 3 \mathrm{H}), 2.35-2.30(\mathrm{~m}, 1 \mathrm{H}), 2.23-2.19(\mathrm{~m}, 1 \mathrm{H}), 1.67(\mathrm{~d}, J=13.0 \mathrm{~Hz}, 1 \mathrm{H}), 1.46-1.43$ $(\mathrm{m}, 1 \mathrm{H}), 1.42(\mathrm{~s}, 9 \mathrm{H}), 1.15-1.05(\mathrm{~m}, 21 \mathrm{H}) \mathrm{ppm} ;{ }^{13} \mathrm{C} \mathrm{NMR}\left(\mathrm{CDCl}_{3}, 125 \mathrm{MHz}\right) \square 153.4,141.2$, $112.4,83.1,82.8,79.6,70.4,59.6,58.0,56.9,47.3,34.9,28.4,18.3,12.1$ ppm. Anal. Calcd for $\mathrm{C}_{24} \mathrm{H}_{45} \mathrm{NO}_{5} \mathrm{Si}$ : C, 63.26; H, 9.95; N, 3.07. Found: C, 63.39; H, 9.84; N, 3.03.

$\mathrm{Me}_{3} \mathrm{SiI}(2.6 \mathrm{~mL}, 18 \mathrm{mmol})$ was added to a solution of this product $(2.10 \mathrm{~g}, 4.61 \mathrm{mmol})$, 2,6-lutidine $(3.5 \mathrm{~mL}, 30 \mathrm{mmol})$ and $\mathrm{CH}_{2} \mathrm{Cl}_{2}(0.11 \mathrm{~L})$ at $0{ }^{\circ} \mathrm{C}$, and the reaction mixture was allowed to warm to room temperature. After $45 \mathrm{~min}, \mathrm{MeOH}(17 \mathrm{~mL})$ was added and the reaction mixture was maintained for an additional $15 \mathrm{~min}$. The reaction then was concentrated in vacuo and crude 14 was used without further purification.

A mixture of 14, paraformaldehyde (3.10 g, corresponds to $103 \mathrm{mmol}$ monomer), toluene $(0.10 \mathrm{~L})$ and $\mathrm{MeCN}(34 \mathrm{~mL})$ was maintained at $80{ }^{\circ} \mathrm{C}$ for $30 \mathrm{~min}$. The reaction mixture was

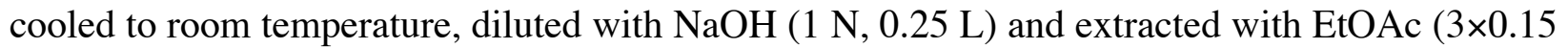
$\mathrm{L})$. The combined organic layers were washed with brine $(0.10 \mathrm{~L})$, dried $\left(\mathrm{MgSO}_{4}\right)$, filtered and the filtrate was concentrated in vacuo. The resulting oil was chromatographed (EtOAc $\square$ 95:5 EtOAc-MeOH) on silica gel to provide $1.60 \mathrm{~g}(4.35 \mathrm{mmol}, 94 \%)$ of $\mathbf{1 5}$ as a colorless oil: IR (film) 2945, 2865, 1730, 1455, 1095, $885 \mathrm{~cm}^{-1}$; ${ }^{1} \mathrm{H}$ NMR $\left(\mathrm{CDCl}_{3}, 500 \mathrm{MHz}\right) \square 3.95(\mathrm{~d}, J=8.0$ $\mathrm{Hz}, 1 \mathrm{H}), 3.68(\mathrm{~d}, J=10.2 \mathrm{~Hz}, 1 \mathrm{H}), 3.58(\mathrm{~d}, J=10.2 \mathrm{~Hz}, 1 \mathrm{H}), 3.45$ (t, $J=5.5 \mathrm{~Hz}, 1 \mathrm{H}), 3.28$ (s, $3 \mathrm{H}), 3.17-3.13(\mathrm{~m}, 1 \mathrm{H}), 3.10-3.04(\mathrm{~m}, 1 \mathrm{H}), 2.97(\mathrm{~d}, J=6.6 \mathrm{~Hz}, 1 \mathrm{H}), 2.51(\mathrm{dd}, J=17.5,5.6$ $\mathrm{Hz}, 1 \mathrm{H}), 2.34-2.20$ (m, $2 \mathrm{H}), 2.22$ (d, $J=17.7 \mathrm{~Hz}, 1 \mathrm{H}), 1.76-1.72(\mathrm{~m}, 1 \mathrm{H}), 1.71$ (d, $J=13.4$ $\mathrm{Hz}, 1 \mathrm{H}), 1.13-1.05(\mathrm{~m}, 21 \mathrm{H}) \mathrm{ppm} ;{ }^{13} \mathrm{C} \mathrm{NMR}\left(\mathrm{CDCl}_{3}, 125 \mathrm{MHz}\right) \square$ 207.8, 83.8, 80.5, 64.9, 60.3, 57.4, 53.4, 46.7, 40.1, 39.3, 30.5, 18.0, 12.1 ppm. Anal. Calcd for $\mathrm{C}_{20} \mathrm{H}_{37} \mathrm{NO}_{3} \mathrm{Si}: \mathrm{C}, 65.35$; H, 10.15; N, 3.81. Found: C, 65.63; H, 10.10; N, 3.57.

Ester 18. To a solution of ester $17(200 \mathrm{mg}, 622 \square \mathrm{mol})$ and $\mathrm{CH}_{2} \mathrm{Cl}_{2}(16 \mathrm{~mL})$ at $-78{ }^{\circ} \mathrm{C}$ was added dropwise a solution of $\mathrm{BBr}_{3}(0.59 \mathrm{~mL}, 6.2 \mathrm{mmol})$ and $\mathrm{CH}_{2} \mathrm{Cl}_{2}(2.0 \mathrm{~mL})$ with vigorous stirring. After keeping the reaction mixture at $-78{ }^{\circ} \mathrm{C}$ for $30 \mathrm{~min}$, it was vigorously stirred at -10 ${ }^{\circ} \mathrm{C}$ for $1.5 \mathrm{~h}$. A solution of $\mathrm{NaOH}$ and brine $(2.14 \mathrm{M}, 11 \mathrm{~mL}, 24 \mathrm{mmol})$ was then added, and the mixture was stirred while slowly warming to room temperature. As soon as all solids were suspended, the mixture was extracted with $\mathrm{CHCl}_{3}(4 \square 20 \mathrm{~mL})$. The combined organic phases were dried $\left(\mathrm{Na}_{2} \mathrm{SO}_{4}\right)$, filtered, and the solvents were removed in vacuo to yield $171 \mathrm{mg}$ (557 $\square \mathrm{mol}, 89 \%$ ) of the corresponding hemiketal as a slightly yellow oil. The crude product was pure 
by ${ }^{1} \mathrm{H}$ NMR analysis and was used without further purification: IR (neat) $3345,2965,1730$, $1450,1375,1320,1275,1175,1035 \mathrm{~cm}^{-1} ;{ }^{1} \mathrm{H}$ NMR $\left(\mathrm{CDCl}_{3}, 400 \mathrm{MHz}\right) \square 5.70(\mathrm{dt}, J=15.5,6.3$ $\mathrm{Hz}, 1 \mathrm{H}), 5.47(\mathrm{dt}, J=15.5,1.4 \mathrm{~Hz}, 1 \mathrm{H}), 4.23$ (br t, $J=2.0 \mathrm{~Hz}, 1 \mathrm{H}), 4.16(\mathrm{qd}, J=7.2,1.3 \mathrm{~Hz}, 2$ H), 3.21-3.16 (br s, $1 \mathrm{H}), 3.08-2.95(\mathrm{~m}, 2 \mathrm{H}), 2.91$ (dd, $J=17.0,9.3 \mathrm{~Hz}, 1 \mathrm{H}), 2.44$ (t, $J=3.4$ $\mathrm{Hz}, 1 \mathrm{H}), 2.23-2.17$ (m, $2 \mathrm{H}), 2.09-2.00$ (m, $2 \mathrm{H}), 1.89-1.78$ (m, $3 \mathrm{H}), 1.63$ (dt, $J=12.2,3.2 \mathrm{~Hz}$, $1 \mathrm{H}), 1.26(\mathrm{t}, J=7.2 \mathrm{~Hz}, 3 \mathrm{H}), 0.97(\mathrm{t}, J=7.4 \mathrm{~Hz}, 3 \mathrm{H}) \mathrm{ppm} ;{ }^{13} \mathrm{C} \mathrm{NMR}\left(\mathrm{CDCl}_{3}, 100 \mathrm{MHz}\right) \square$ 175.5, 132.6, 126.9, 104.8, 82.1, 81.6, 65.2, 61.5, 55.9, 47.7, 36.8, 33.1, 32.4, 26.6, 25.3, 14.1, 13.5 ppm; HRMS (CI) $\mathrm{m} / z 307.1779$ (calcd for $\mathrm{C}_{17} \mathrm{H}_{25} \mathrm{NO}_{4} 307.1783$ ).

A solution of this hemiketal $(164 \mathrm{mg}, 533 \square \mathrm{mol})$ and (trimethylsilyl)imidazole $(3.0 \mathrm{~mL}$, $20 \mathrm{mmol}$ ) was heated to $130{ }^{\circ} \mathrm{C}$ for $20 \mathrm{~min}$. The reaction mixture was then cooled to $0{ }^{\circ} \mathrm{C}$, EtOAc and hexanes (50:50, $10 \mathrm{~mL}$ ) were added, and the mixture was chromatographed (50:50 EtOAc-hexanes) immediately on silica gel (treated with $\mathrm{NEt}_{3}$ ) to provide $156 \mathrm{mg}$ (411 mmol, $77 \%$ ) of the corresponding silyl ketal as a slightly yellow oil: IR (neat) 2960, 1735, 1455, 1375, 1330, 1295, 1225, 1160, $1035 \mathrm{~cm}^{-1}$; ${ }^{1} \mathrm{H}$ NMR $\left(\mathrm{CDCl}_{3}, 400 \mathrm{MHz}\right) \square 5.65(\mathrm{dt}, J=15.5,6.3 \mathrm{~Hz}, 1$ H), 5.44 (dt, $J=15.5,1.4 \mathrm{~Hz}, 1 \mathrm{H}), 4.20-4.16$ (br s, $1 \mathrm{H}), 4.16-4.02$ (m, $2 \mathrm{H}$ ), 3.21-3.16 (br s, 1 H), 3.05-2.95 (m, $2 \mathrm{H}), 2.67(\mathrm{dd}, J=15.9,6.5 \mathrm{~Hz}, 1 \mathrm{H}), 2.35(\mathrm{~d}, J=4.8 \mathrm{~Hz}, 1 \mathrm{H}), 2.33-2.27(\mathrm{~m}$, $1 \mathrm{H}), 2.10(\mathrm{dd}, J=15.9,6.9 \mathrm{~Hz}, 1 \mathrm{H}), 2.07-1.99(\mathrm{~m}, 2 \mathrm{H}), 1.89-1.73(\mathrm{~m}, 3 \mathrm{H}), 1.57$ (dt, $J=11.9$, $3.0 \mathrm{~Hz}, 1 \mathrm{H}), 1.23(\mathrm{t}, J=7.1 \mathrm{~Hz}, 3 \mathrm{H}), 0.96(\mathrm{t}, J=7.4 \mathrm{~Hz}, 3 \mathrm{H}), 0.12(\mathrm{~s}, 9 \mathrm{H}) \mathrm{ppm} ;{ }^{13} \mathrm{C}$ NMR $\left(\mathrm{CDCl}_{3}, 100 \mathrm{MHz}\right) \square 173.2,132.1,127.6,106.5,82.0,81.8,64.0,60.2,56.9,47.9,37.7,33.0$, 31.6, 26.8, 25.3, 14.2, 13.5, $1.7 \mathrm{ppm}$; HRMS (CI) $\mathrm{m} / z 379.2165$ (calcd for $\mathrm{C}_{20} \mathrm{H}_{33} \mathrm{NO}_{4} \mathrm{Si}$ 379.2179).

To a solution of this silyl ketal ester (414 mg, $1.09 \mathrm{mmol})$, DMPU (3.3 mL, $27 \mathrm{mmol}$ ) and THF $(16 \mathrm{~mL})$ at $-45^{\circ} \mathrm{C}$ was added dropwise a freshly prepared solution of LDA in THF $(0.4$ $\mathrm{M}, 4.9 \mathrm{~mL}, 2.0 \mathrm{mmol})$. After stirring for $1 \mathrm{~h}$ at $-45^{\circ} \mathrm{C}$, MeI $(0.54 \mathrm{~mL}, 8.7 \mathrm{mmol})$ was added and the reaction was maintained for $1 \mathrm{~h}$ at this temperature. A solution of DABCO $(978 \mathrm{mg}, 8.72$ $\mathrm{mmol})$ and THF $(4.0 \mathrm{~mL})$ was then added and stirring was continued for $30 \mathrm{~min}$ at $-45^{\circ} \mathrm{C}$. After the addition of brine $(15 \mathrm{~mL})$, the mixture was allowed to warm to room temperature. The mixture was extracted with $\mathrm{CHCl}_{3}(3 \square 40 \mathrm{~mL})$, the combined organic phases were dried $\left(\mathrm{Na}_{2} \mathrm{SO}_{4}\right)$, filtered, and the solvents were removed in vacuo. Chromatography (33:67 EtOAc-hexanes) of the crude product on silica gel afforded $338 \mathrm{mg}(859 \square \mathrm{mol}, 79 \%)$ of 18 as a slightly yellow oil: IR (neat) 2690, 1735, 1460, 1375, 1325, 1250, 1225, 1155, $1040 \mathrm{~cm}^{-1} ;{ }^{1} \mathrm{H}$ NMR $\left(\mathrm{CDCl}_{3}, 500 \mathrm{MHz}\right) \square 5.66(\mathrm{dt}, J=15.5,6.3 \mathrm{~Hz}, 1 \mathrm{H}), 5.44(\mathrm{~d}, J=15.5 \mathrm{~Hz}, 1 \mathrm{H}), 4.23-4.19$ (br s, 1 H), 4.19-4.11 (m, 1 H), 4.11-4.03 (m, 1 H), 3.18-3.14 (br s, $1 \mathrm{H}), 2.97$ (t, J = 7.3 Hz, 2 H), 2.82-2.75 (m, $1 \mathrm{H}), 2.33(\mathrm{~d}, J=5.4 \mathrm{~Hz}, 1 \mathrm{H}), 2.08-2.01(\mathrm{~m}, 2 \mathrm{H}), 1.97(\mathrm{~d}, J=12.0 \mathrm{~Hz}, 1 \mathrm{H})$, 1.86-1.75 (m, $3 \mathrm{H}), 1.54(\mathrm{dt}, J=12.0,3.2 \mathrm{~Hz}, 1 \mathrm{H}), 1.35(\mathrm{~d}, J=6.7 \mathrm{~Hz}, 3 \mathrm{H}), 1.23(\mathrm{t}, J=7.1 \mathrm{~Hz}$, $3 \mathrm{H}), 0.97(\mathrm{t}, J=7.4 \mathrm{~Hz}, 3 \mathrm{H}), 0.16(\mathrm{~s}, 9 \mathrm{H}) \mathrm{ppm} ;{ }^{13} \mathrm{C} \mathrm{NMR}\left(\mathrm{CDCl}_{3}, 125 \mathrm{MHz}\right) \square 176.1,132.2$, 127.6, 107.8, 82.5, 81.6, 63.5, 60.1, 57.9, 47.8, 43.8, 39.0, 33.8, 26.8, 25.3, 19.5, 14.2, 13.5, 1.8 ppm; HRMS (CI) $\mathrm{m} / \mathrm{z} 393.2344$ (calcd for $\mathrm{C}_{21} \mathrm{H}_{35} \mathrm{NO}_{4} \mathrm{Si} 393.2335$ ).

Tetronate addition to aldehyde 20 to give 21. To a solution of 4-methoxy-3-methyl$2(5 \mathrm{H})$ furanone $(\mathbf{2 5})^{10}(181 \mathrm{mg}, 1.41 \mathrm{mmol})$ and THF $(4.5 \mathrm{~mL})$ at $-78{ }^{\circ} \mathrm{C}$ was added dropwise a solution of $n$-butyllithium in hexanes $(3.31 \mathrm{M}, 0.34 \mathrm{~mL}, 1.13 \mathrm{mmol})$. After stirring for $1 \mathrm{~h}$ at -78 ${ }^{\circ} \mathrm{C}$, a solution of aldehyde $20(99.0 \mathrm{mg}, 283 \square \mathrm{mol})$ and THF $(0.50 \mathrm{~mL})$ was added, and stirring was continued at this temperature for $1 \mathrm{~h}$. After addition of brine $(8.0 \mathrm{~mL})$, the mixture was

\footnotetext{
${ }^{10}$ Knight, D. W.; Pattenden, G. J. Chem. Soc., Perkin Trans. 1 1975, 635.
} 
allowed to warm to room temperature and was extracted with $\mathrm{CHCl}_{3}$ (4 $\square 25 \mathrm{~mL}$ ). The combined organic phases were dried $\left(\mathrm{Na}_{2} \mathrm{SO}_{4}\right)$, filtered, and the solvents were removed in vacuo. Chromatography (EtOAc $\square \quad$ 98:2 EtOAc-MeOH $\square \quad$ 95:5 EtOAc-MeOH $\square \quad$ 90:10 EtOAc-MeOH) of the residue afforded $127 \mathrm{mg}(266 \square \mathrm{mol}, 94 \%)$ of the corresponding adduct (a 59:22:19 mixture of isomers according to ${ }^{1} \mathrm{H}$ NMR analysis) as a colorless residue. Spectroscopic data for this mixture of isomers: IR (neat) 3450, 2960, 1750, 1665, 1455, 1390, $1335,1250,1225,1145,1090 \mathrm{~cm}^{-1} ;{ }^{1} \mathrm{H} \mathrm{NMR}\left(\mathrm{CDCl}_{3}, 500 \mathrm{MHz}\right)$ is very complex and was not analyzed in detail, see reproduction; ${ }^{13} \mathrm{C} \mathrm{NMR}\left(\mathrm{CDCl}_{3}, 100 \mathrm{MHz}\right) \square 175.3,175.1,174.3,174.1$, 171.9, 171.8, 132.4, 132.2, 132.1, 127.7, 127.7, 127.5, 108.3, 107.7, 98.0, 97.6, 97.4, 82.0, 82.0, $81.9,81.9,81.8,80.2,77.7,74.2,73.9,72.2,63.5,63.4,63.2,59.3,58.7,57.6,57.3,57.0,48.0$, 47.9, 47.9, 39.8, 39.7, 39.4, 35.6, 34.7, 34.6, 34.3, 32.5, 31.5, 26.9, 25.3, 22.3, 14.3, 14.0, 13.5, $10.7,9.7,8.6,8.5,8.5,1.8,1.7,1.5 \mathrm{ppm}$; HRMS (CI) $\mathrm{m} / z$ 477.2549 (calcd for $\mathrm{C}_{25} \mathrm{H}_{39} \mathrm{NO}_{6} \mathrm{Si}$ 477.2547).

To a solution of this intermediate $(127 \mathrm{mg}, 266 \square \mathrm{mol}), \mathrm{MeOH}(4.0 \mathrm{~mL})$ and $\mathrm{CHCl}_{3}(0.70$ $\mathrm{mL})$ was added aqueous $\mathrm{HCl}(1 \mathrm{~N}, 1.0 \mathrm{~mL}, 1.0 \mathrm{mmol})$. After $15 \mathrm{~h}, \mathrm{~K}_{2} \mathrm{CO}_{3}(3.4 \mathrm{~g})$ was added and the suspension was stirred vigorously for $1 \mathrm{~h}$. The solvents then were removed in vacuo, $\mathrm{CHCl}_{3}$ $(20 \mathrm{~mL})$ was added and the suspension was stirred for $1 \mathrm{~h}$. The solids were removed by filteration and the filtrate was concentrated to give $108 \mathrm{mg}$ (266 $\square \mathrm{mol}, 99 \%)$ of diols 21 as a colorless oil. Spectroscopic data for this mixture of isomers: IR (neat) 3220, 2960, 1745, 1665, $1455,1390,1335,1225,1095 \mathrm{~cm}^{-1} ;{ }^{1} \mathrm{H} \mathrm{NMR}\left(\mathrm{CDCl}_{3}, 500 \mathrm{MHz}\right)$ is very complex and was not analyzed in detail, see reproduction; ${ }^{13} \mathrm{C} \mathrm{NMR}\left(\mathrm{CDCl}_{3}, 125 \mathrm{MHz}\right) \square 175.6,175.2,172.0,171.8$, $132.8,126.8,105.8,98.2,82.0,78.4,78.3,74.0,72.4,65.0,64.0,63.6,59.5,59.0,58.9,57.2$, 57.0, 47.6, 40.5, 35.3, 34.9, 34.0, 33.2, 29.7, 26.5, 25.4, 14.9, 14.0, 13.5, 8.5 ppm; HRMS (CI) $\mathrm{m} / \mathrm{z} 405.2142$ (calcd for $\mathrm{C}_{22} \mathrm{H}_{31} \mathrm{NO}_{6} 405.2151$ ).

IBX oxidation of 21 to give diol 22. A solution of diols 21 (50.0 mg, $123 \square \mathrm{mol})$, IBX (276 mg, $986 \square \mathrm{mol}$ ) and DMSO (2.0 mL) was heated to $55^{\circ} \mathrm{C}$ for $6 \mathrm{~h} .{ }^{11}$ After cooling to room temperature, saturated aqueous $\mathrm{NaHCO}_{3}(2.0 \mathrm{~mL})$ was added and stirring was continued for $2 \mathrm{~h}$. The mixture then was extracted with $\mathrm{CHCl}_{3}(5 \square 30 \mathrm{~mL})$, the combined organic phases were dried $\left(\mathrm{Na}_{2} \mathrm{SO}_{4}\right)$ and the solvents were removed in vacuo. Chromatography (98:2 EtOAc-MeOH) of this residue afforded $28.4 \mathrm{mg}(67.7 \square \mathrm{mol}, 55 \%)$ of diol 22 as a colorless oil that was mainly one isomer of unknown configuration (NMR analysis): IR (neat) 3355, 2960, 1755, 1675, 1455, $1390,1325,1275,1130,1020 \mathrm{~cm}^{-1} ;{ }^{1} \mathrm{H} \mathrm{NMR}\left(\mathrm{CDCl}_{3}, 500 \mathrm{MHz}\right)$ is very complex and was not analyzed in detail, see reproduction; ${ }^{13} \mathrm{C} \mathrm{NMR}\left(\mathrm{CDCl}_{3}, 125 \mathrm{MHz}\right) \square 172.6,167.8,133.4,133.3$, 126.4, 101.7, 99.9, 83.2, 80.8, 60.9, 60.8, 60.4, 58.9, 51.0, 47.8, 45.9, 35.9, 32.5, 29.6, 26.4, 25.3, 21.0, 14.1, 13.4, 12.4, 8.5 ppm; HRMS (CI) $m / z 419.1939$ (calcd for $\mathrm{C}_{22} \mathrm{H}_{29} \mathrm{NO}_{7} 419.1944$ ).

Thionocarbonates 23 and 24. A solution of diol 22 (18.0 mg, $42.9 \square \mathrm{mol})$ and $\mathrm{CH}_{2} \mathrm{Cl}_{2}$ $(0.50 \mathrm{~mL})$ was cooled $-50{ }^{\circ} \mathrm{C}$ and a solution of DMAP $(63.0 \mathrm{mg}, 516 \square \mathrm{mol})$ and $\mathrm{CH}_{2} \mathrm{Cl}_{2}(1.0$ $\mathrm{mL})$ was added. A solution of thiophosgene in $\mathrm{CCl}_{4}(1.5 \mathrm{M}, 0.17 \mathrm{~mL}, 0.25 \mathrm{mmol})$ then was added slowly over 30 min. ${ }^{12}$ Stirring at $-50{ }^{\circ} \mathrm{C}$ was continued for $2 \mathrm{~h}$; saturated aqueous $\mathrm{NaHCO}_{3}$ $(3.0 \mathrm{~mL})$ was added and the mixture was allowed to warm to room temperature. After extraction with $\mathrm{CHCl}_{3}(3 \square 15 \mathrm{~mL})$, the combined organic phases were dried $\left(\mathrm{Na}_{2} \mathrm{SO}_{4}\right)$ and the solvents were

\footnotetext{
${ }^{11}$ Frigerio, M.; Santagostino, M. Tetrahedron Lett. 1994, 35, 8019.

${ }^{12}$ Corey, E. J.; Hopkin, P. B. Tetrahedron Lett. 1982, 23, 1979.
} 
removed in vacuo. Chromatography (98.5:1.5 $\left.\mathrm{CHCl}_{3}-\mathrm{MeOH}\right)$ of the residue gave $2.6 \mathrm{mg}$ (5.6 $\square \mathrm{mol}, 13 \%)$ of the high- $\mathrm{R}_{\mathrm{f}}$ diastereomer 24 and $9.7 \mathrm{mg}(21 \square \mathrm{mol}, 49 \%)$ of the low- $\mathrm{R}_{\mathrm{f}}$ diastereomer 23. Characterization data for 24: IR (neat) 2960, 1795, 1680, 1455, 1390, 1335, 1270, 1210, 1130, 1025, $990 \mathrm{~cm}^{-1} ;{ }^{1} \mathrm{H} \mathrm{NMR}\left(\mathrm{CDCl}_{3}, 500 \mathrm{MHz}\right) \square 5.75(\mathrm{dt}, J=15.5,6.4 \mathrm{~Hz}, 1$ H), $5.46(\mathrm{~d}, J=15.5 \mathrm{~Hz}, 1 \mathrm{H}), 4.16(\mathrm{~s}, 3 \mathrm{H}), 4.14-4.12($ br s, $1 \mathrm{H}), 3.47-3.42($ br s, $1 \mathrm{H})$, 3.14-3.05 (m, $1 \mathrm{H}), 3.01-2.93(\mathrm{~m}, 1 \mathrm{H}), 2.79-2.71(\mathrm{~m}, 1 \mathrm{H}), 2.69(\mathrm{~d}, J=5.2 \mathrm{~Hz}, 1 \mathrm{H}), 2.11-2.03$ $(\mathrm{m}, 2 \mathrm{H}), 2.04(\mathrm{~s}, 3 \mathrm{H}), 1.91(\mathrm{dd}, J=12.3,3.5 \mathrm{~Hz}, 1 \mathrm{H}), 1.87-1.78(\mathrm{~m}, 3 \mathrm{H}), 1.75-1.69(\mathrm{~m}, 1 \mathrm{H})$, $1.22(\mathrm{~d}, J=6.7 \mathrm{~Hz}, 3 \mathrm{H}), 0.99(\mathrm{t}, J=7.5 \mathrm{~Hz}, 3 \mathrm{H}) \mathrm{ppm} ;{ }^{13} \mathrm{C} \mathrm{NMR}\left(\mathrm{CDCl}_{3}, 125 \mathrm{MHz}\right) \square 185.9$, 169.1, 165.1, 133.3, 126.4, 118.4, 113.4, 106.6, 101.8, 82.3, 80.9, 60.4, 59.8, 51.4, 48.0, 43.6, 38.1, 32.4, 26.6, 25.3, 13.4, 11.0, $8.3 \mathrm{ppm}$; HRMS (ESI) $\mathrm{m} / \mathrm{z} 462.1596$ (calcd for $\mathrm{C}_{23} \mathrm{H}_{27} \mathrm{NO}_{7} \mathrm{~S}+\mathrm{H}^{+}$462.1587). Characterization data for 23: IR (neat) 2960, 1795, 1680, 1455, 1390, 1335, 1270, 1210, $990 \mathrm{~cm}^{-1} ;{ }^{1} \mathrm{H}$ NMR $\left(\mathrm{CDCl}_{3}, 500 \mathrm{MHz}\right) \square 5.74(\mathrm{dt}, J=15.5,6.4 \mathrm{~Hz}, 1$ H), $5.44(\mathrm{dt}, J=15.5,1.5 \mathrm{~Hz}, 1 \mathrm{H}), 4.22$ (s, $3 \mathrm{H}), 4.16-4.14$ (br s, $1 \mathrm{H}), 3.47-3.41$ (br s, $1 \mathrm{H})$, 3.13-3.04 (m, $1 \mathrm{H}), 3.00-2.92(\mathrm{~m}, 1 \mathrm{H}), 2.78(\mathrm{~d}, J=5.2 \mathrm{~Hz}, 1 \mathrm{H}), 2.69-2.60(\mathrm{~m}, 1 \mathrm{H}), 2.10(\mathrm{~s}, 3$ H), 2.10-2.03 (m, $2 \mathrm{H}), 1.95(\mathrm{dd}, J=11.9,3.7 \mathrm{~Hz}, 1 \mathrm{H}), 1.89-1.81(\mathrm{~m}, 2 \mathrm{H}), 1.80(\mathrm{~d}, J=12.1$ $\mathrm{Hz}, 1 \mathrm{H}), 1.73(\mathrm{dt}, J=12.2,3.4 \mathrm{~Hz}, 1 \mathrm{H}), 1.21(\mathrm{~d}, J=6.7 \mathrm{~Hz}, 3 \mathrm{H}), 0.98(\mathrm{t}, J=7.4 \mathrm{~Hz}, 3 \mathrm{H})$ ppm; ${ }^{13} \mathrm{C} \mathrm{NMR}\left(\mathrm{CDCl}_{3}, 125 \mathrm{MHz}\right) \square 185.5,168.2,162.6,133.4,126.4,117.0,112.7,105.9$, $102.9,82.4,80.7,60.4,59.8,51.3,48.0,44.7,39.3,32.5,26.5,25.3,13.5,11.3,8.6$ ppm; HRMS (CI) $\mathrm{m} / z, 461.1518$ (calcd for $\mathrm{C}_{23} \mathrm{H}_{27} \mathrm{NO}_{7} \mathrm{~S} 461.1508$ ).

Didehydrostemofoline (1). A solution of thiocarbonate 23 (14.0 mg, $30.3 \square \mathrm{mol})$ and $\mathrm{P}(\mathrm{OMe})_{3}(1.0 \mathrm{~mL}, 8.48 \mathrm{mmol})$ was heated to $120^{\circ} \mathrm{C}$ in a sealed tube for $12 \mathrm{~h} .{ }^{13}$ After cooling to room temperature, the mixture was diluted with EtOAc $(20 \mathrm{~mL})$ and washed with saturated aqueous $\mathrm{NaHCO}_{3}(4.0 \mathrm{~mL})$. The phases were separated and the aqueous layer was extracted with EtOAc $(3 \square 20 \mathrm{~mL})$. The combined organic layers were dried $\left(\mathrm{Na}_{2} \mathrm{SO}_{4}\right)$ and the solvents and $\mathrm{P}(\mathrm{OMe})_{3}$ were removed in vacuo. The ${ }^{1} \mathrm{H}$ NMR spectrum of the crude product showed the desired product 1 and also $15 \%$ of isodidehydrostemofoline 3. Chromatography of this residue (50:50 EtOAc:hexanes $\square$ EtOAc) gave $7.7 \mathrm{mg}(20 \square \mathrm{mol}, 66 \%)$ of pure didehydrostemofoline (1), which was indistinguishable, with the exception of optical rotation, from an authentic specimen kindly provided by Professor Suratwadee Jiwajinda, Kasetsart University, Thailand.

Isodidehydrostemofoline 3. Thiocarbonate $24(3.0 \mathrm{mg}, 6.5 \square \mathrm{mol})$ in $\mathrm{P}(\mathrm{OMe})_{3}(0.50 \mathrm{~mL}$, $4.24 \mathrm{mmol}$ ) was heated to $120^{\circ} \mathrm{C}$ in a sealed tube for $12 \mathrm{~h}$. After cooling to room temperature, the mixture was diluted with EtOAc $(10 \mathrm{~mL})$ and washed with saturated aqueous $\mathrm{NaHCO}_{3}(2.0$ $\mathrm{mL})$. The phases were separated and the aqueous layer was extracted with EtOAc $(3 \square 20 \mathrm{~mL})$. The combined organic layers were dried $\left(\mathrm{Na}_{2} \mathrm{SO}_{4}\right)$ and the solvents and $\mathrm{P}(\mathrm{OMe})_{3}$ were removed in vacuo. The ${ }^{1} \mathrm{H}$ NMR spectrum of the crude product showed the desired product 3 and traces of didehydrostemofoline 1. Chromatography of this residue (50:50 EtOAc:hexanes $\square$ EtOAc) gave $1.6 \mathrm{mg}$ (4.2 Ømol, 64\%) of pure isodidehydrostemofoline (3), which showed NMR data indistinguishable from those of the natural product (data kindly provided by Professor Suratwadee Jiwajinda, Kasetsart University, Thailand).

\footnotetext{
${ }^{13}$ Corey, E. J.; Carey, F. A.; Winter, R. A. E. J. Am. Chem. Soc. 1965, 87, 934.
} 

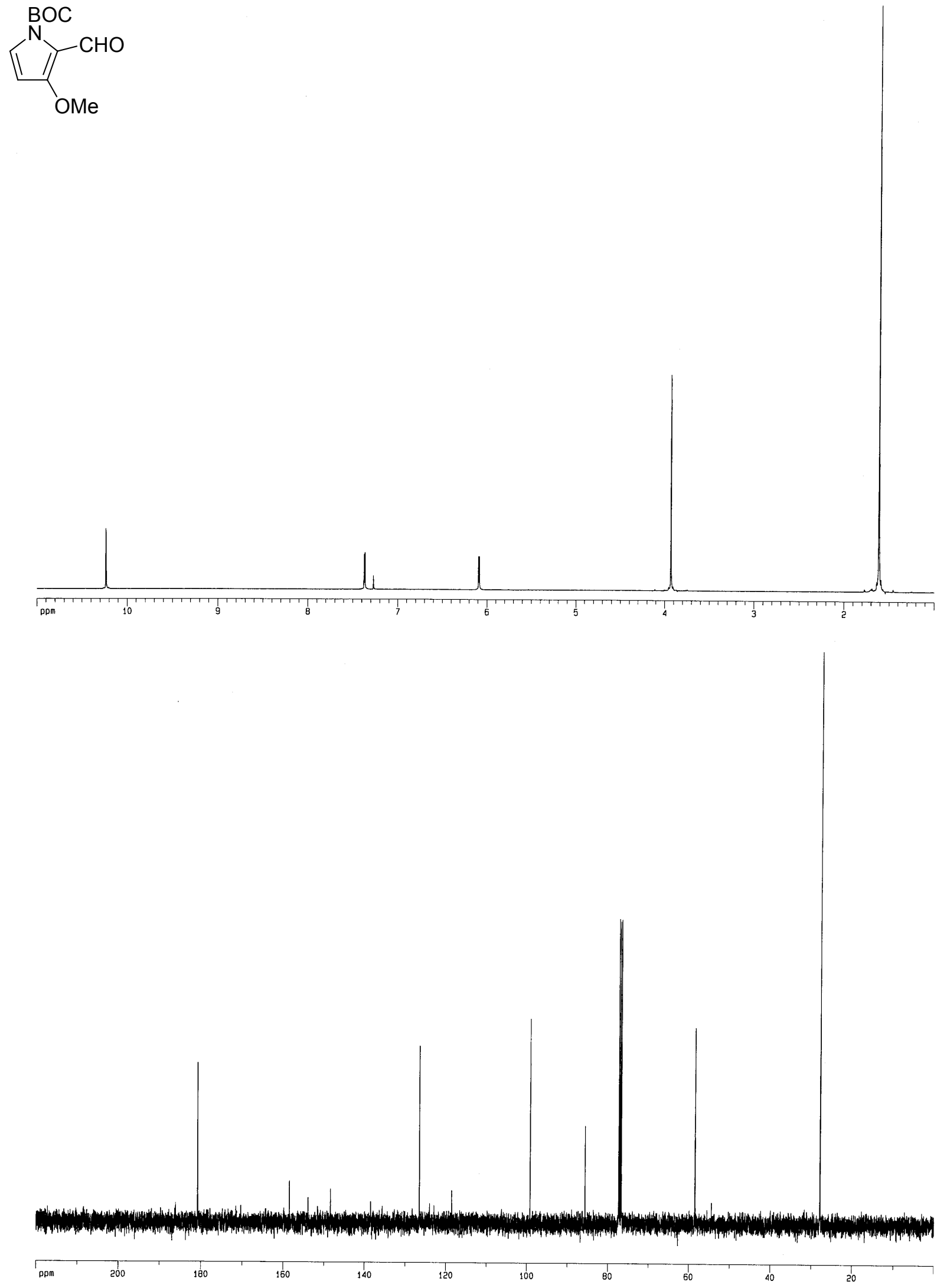
S31
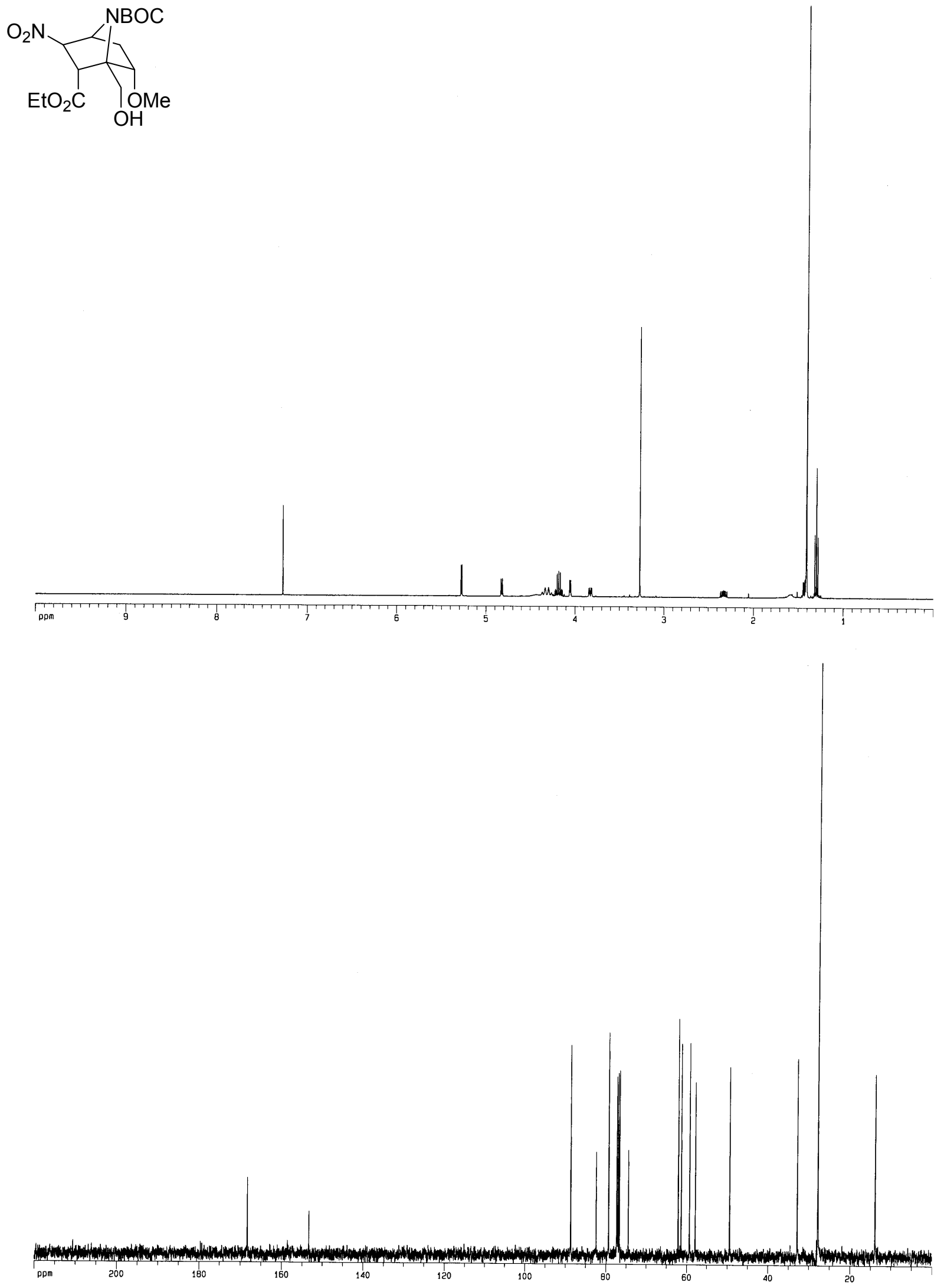

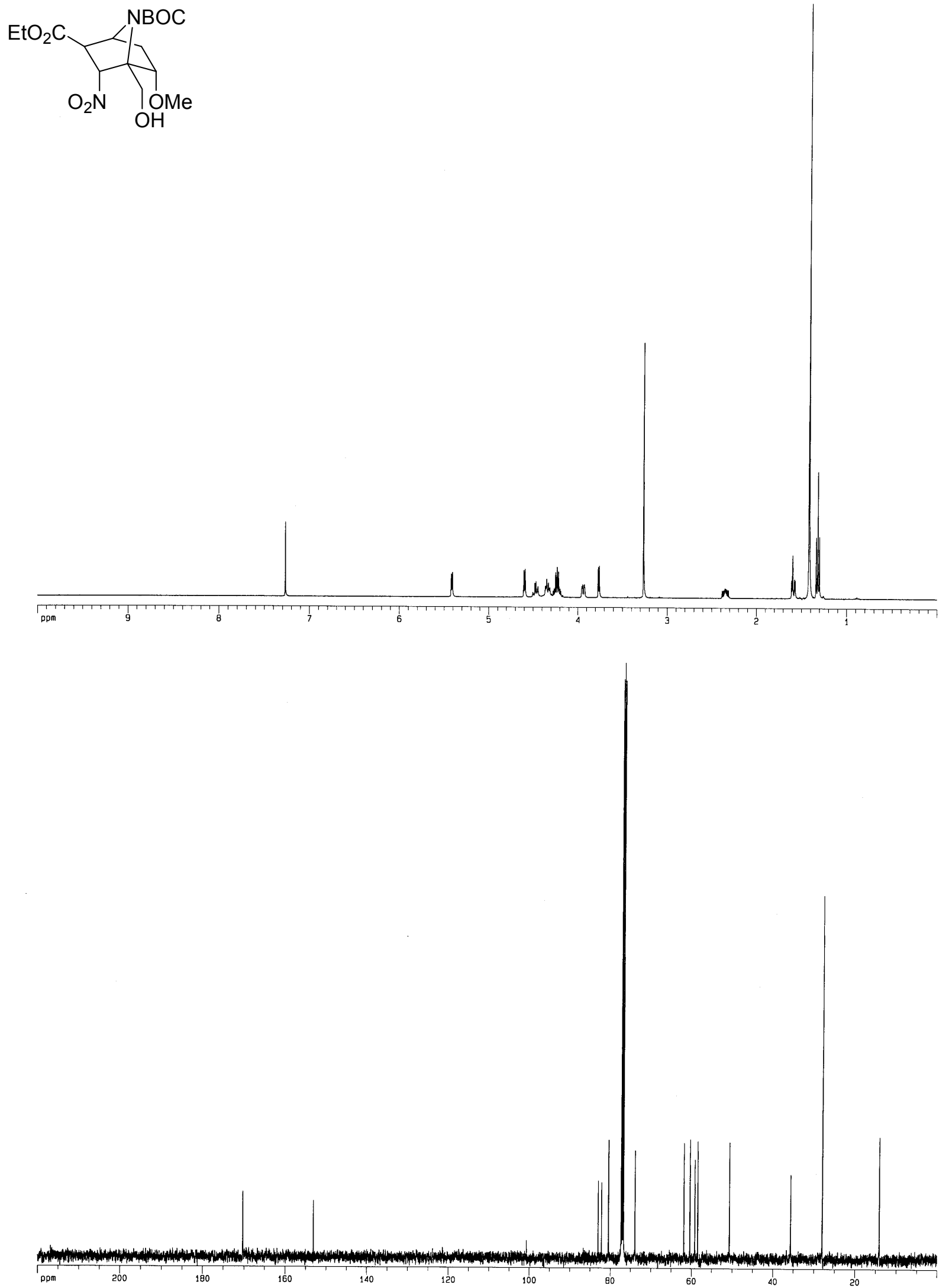

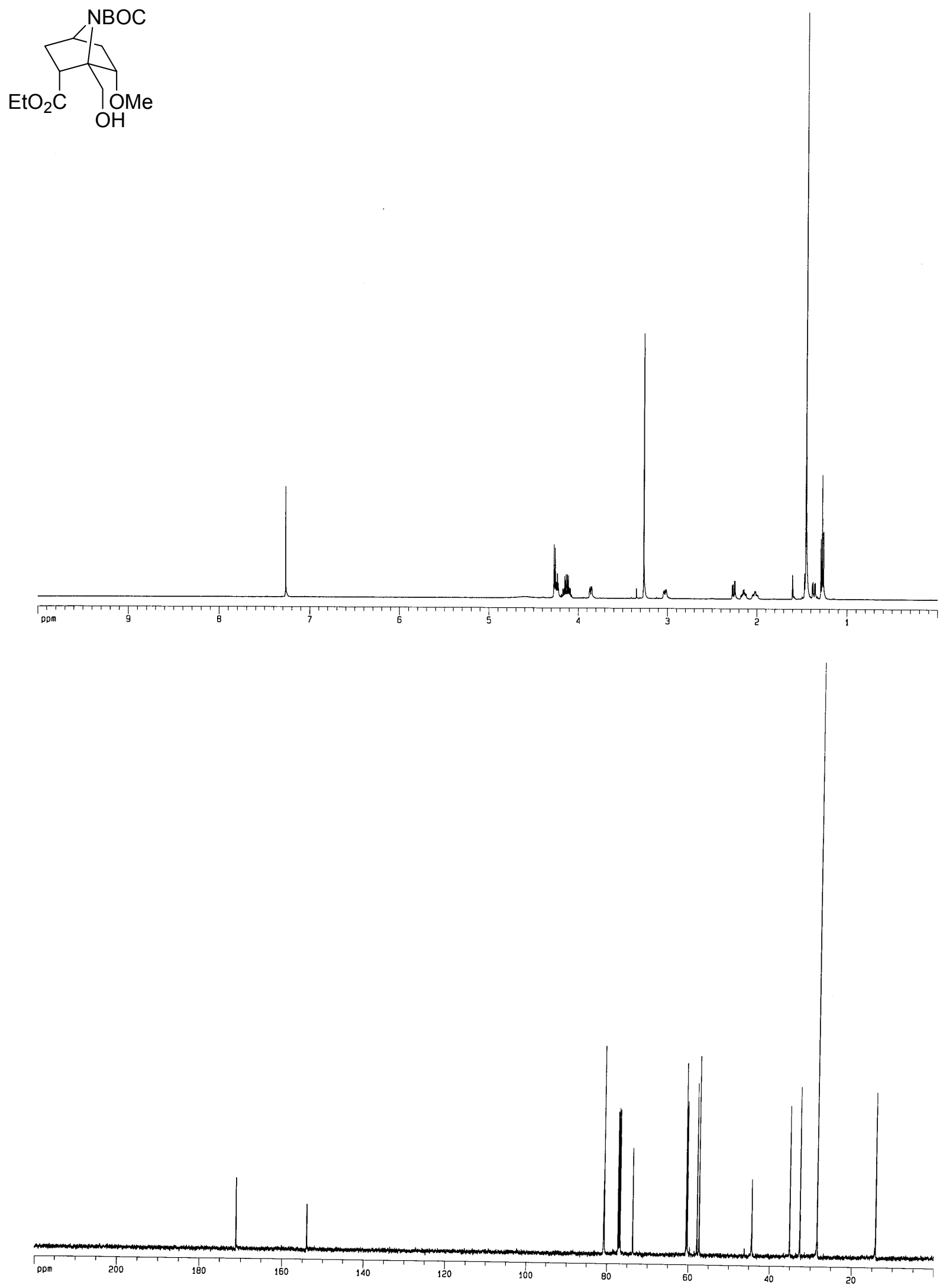
S11
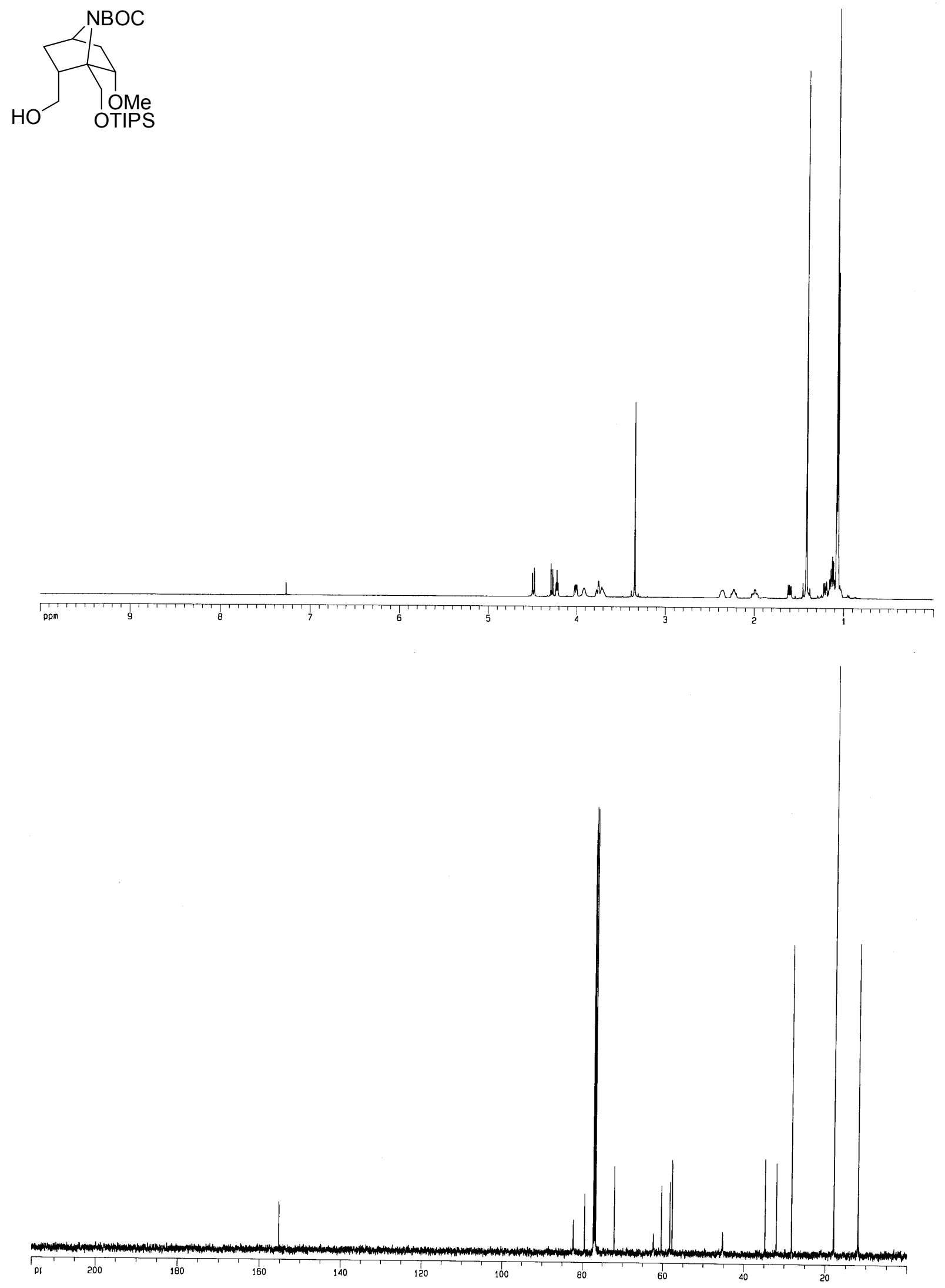
S12
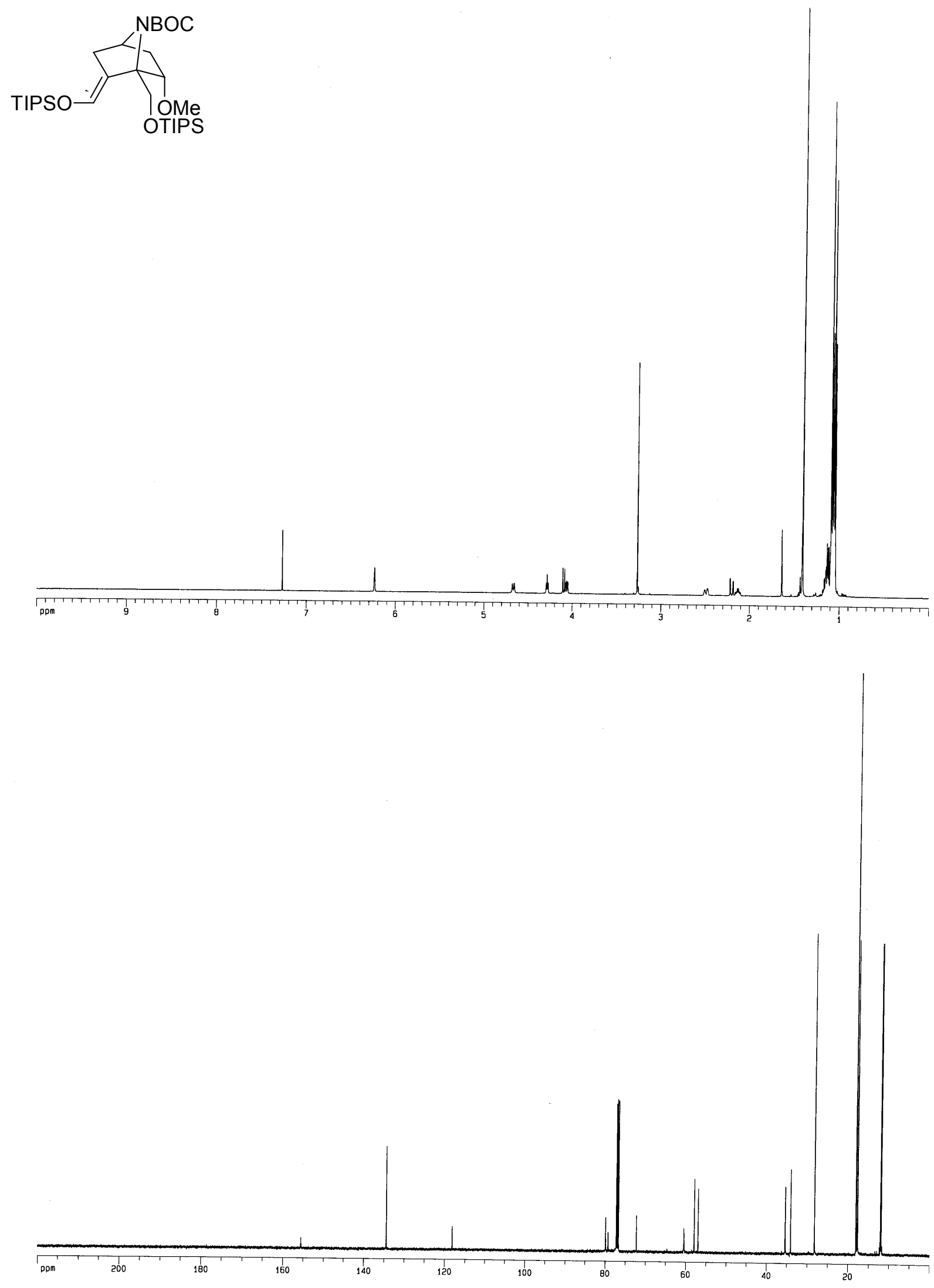
S13
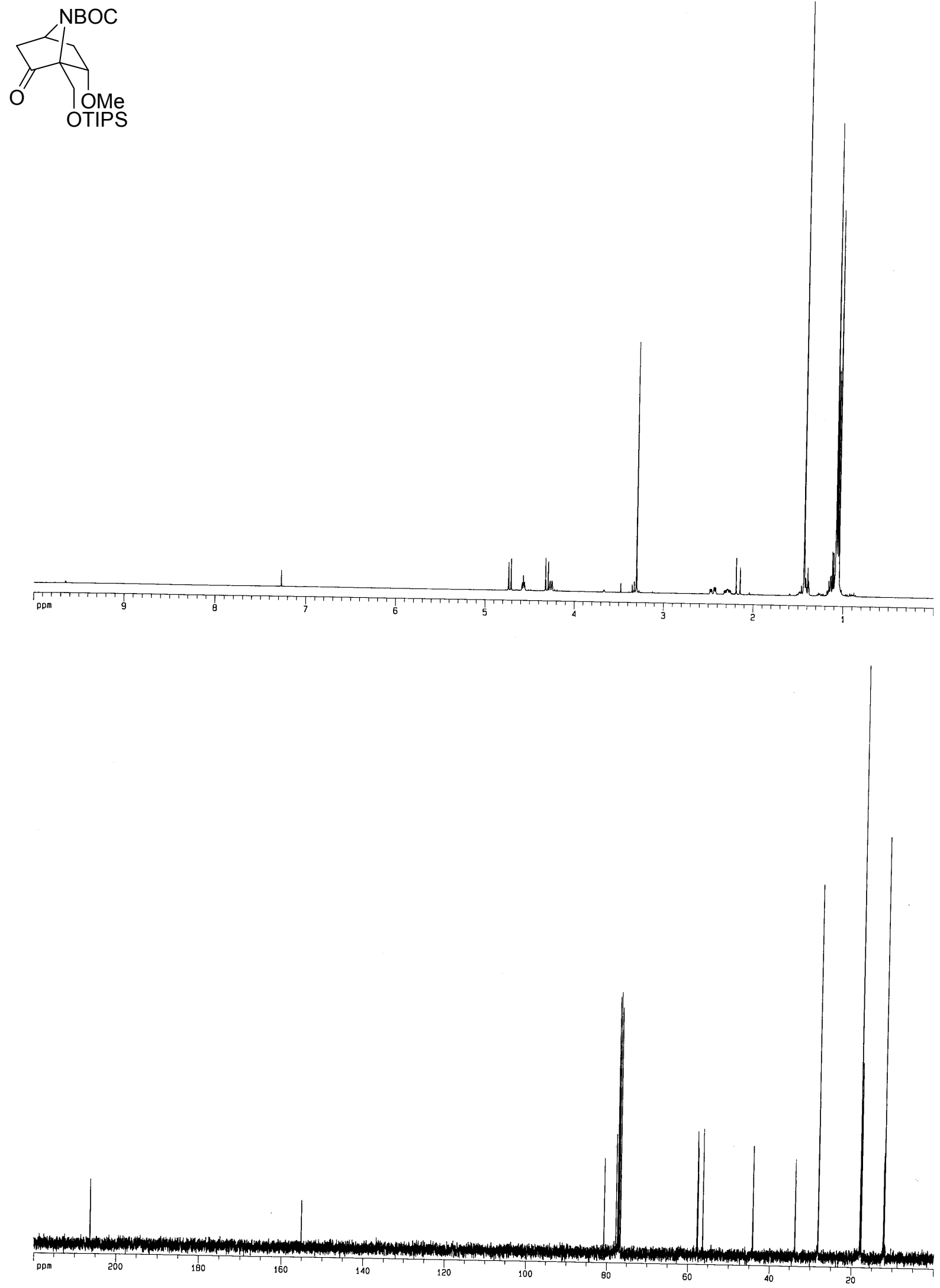

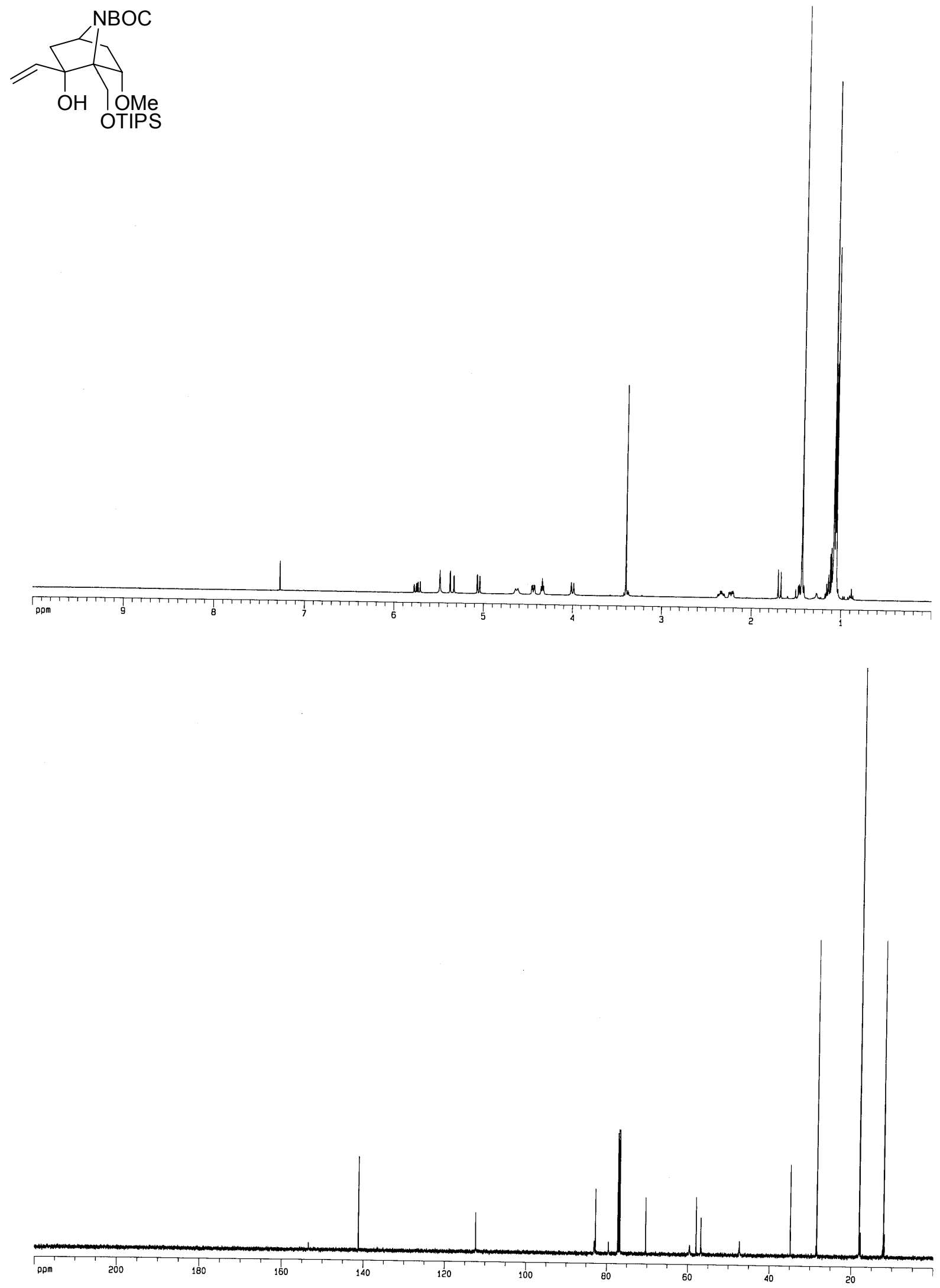

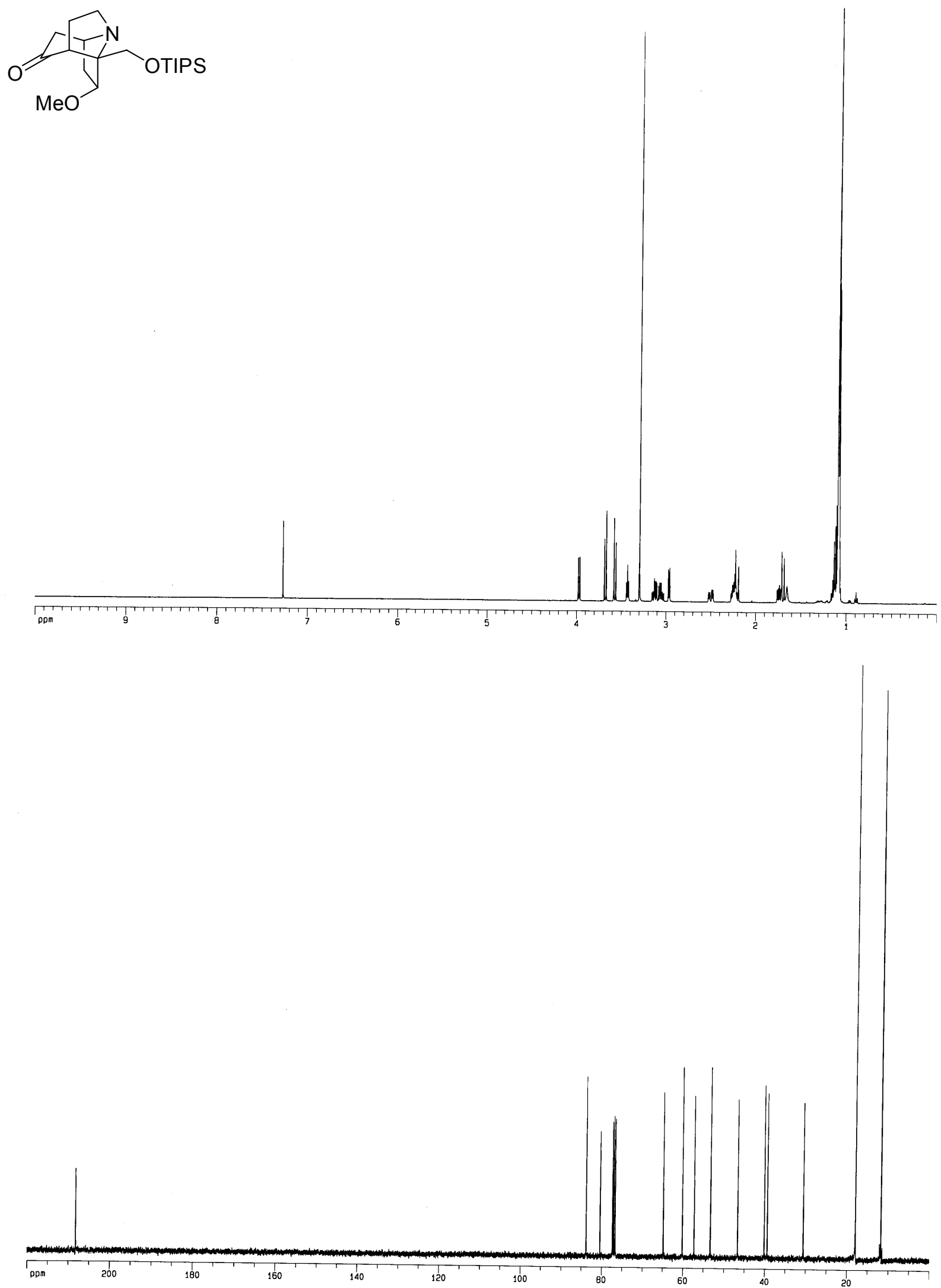
S16
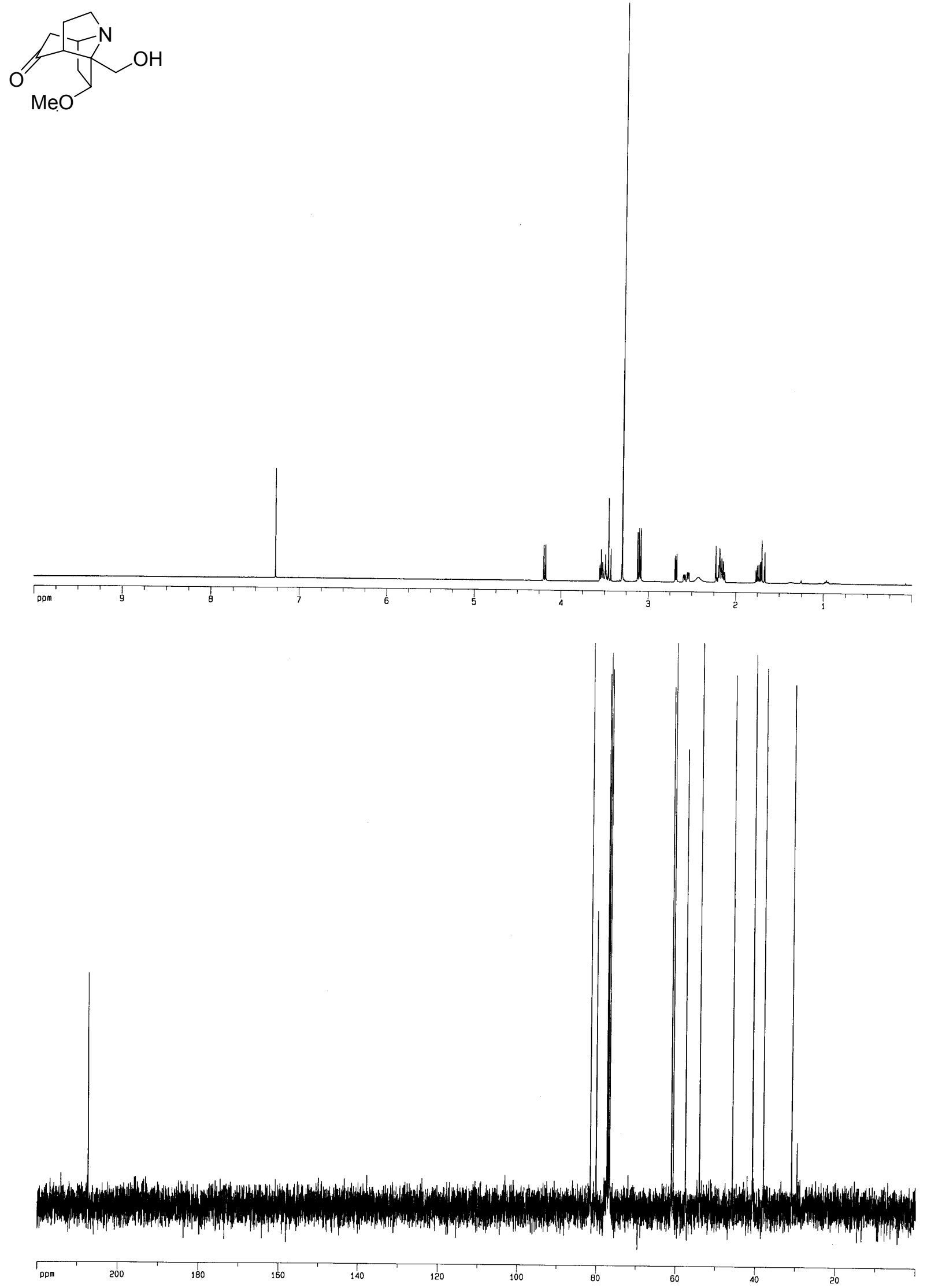

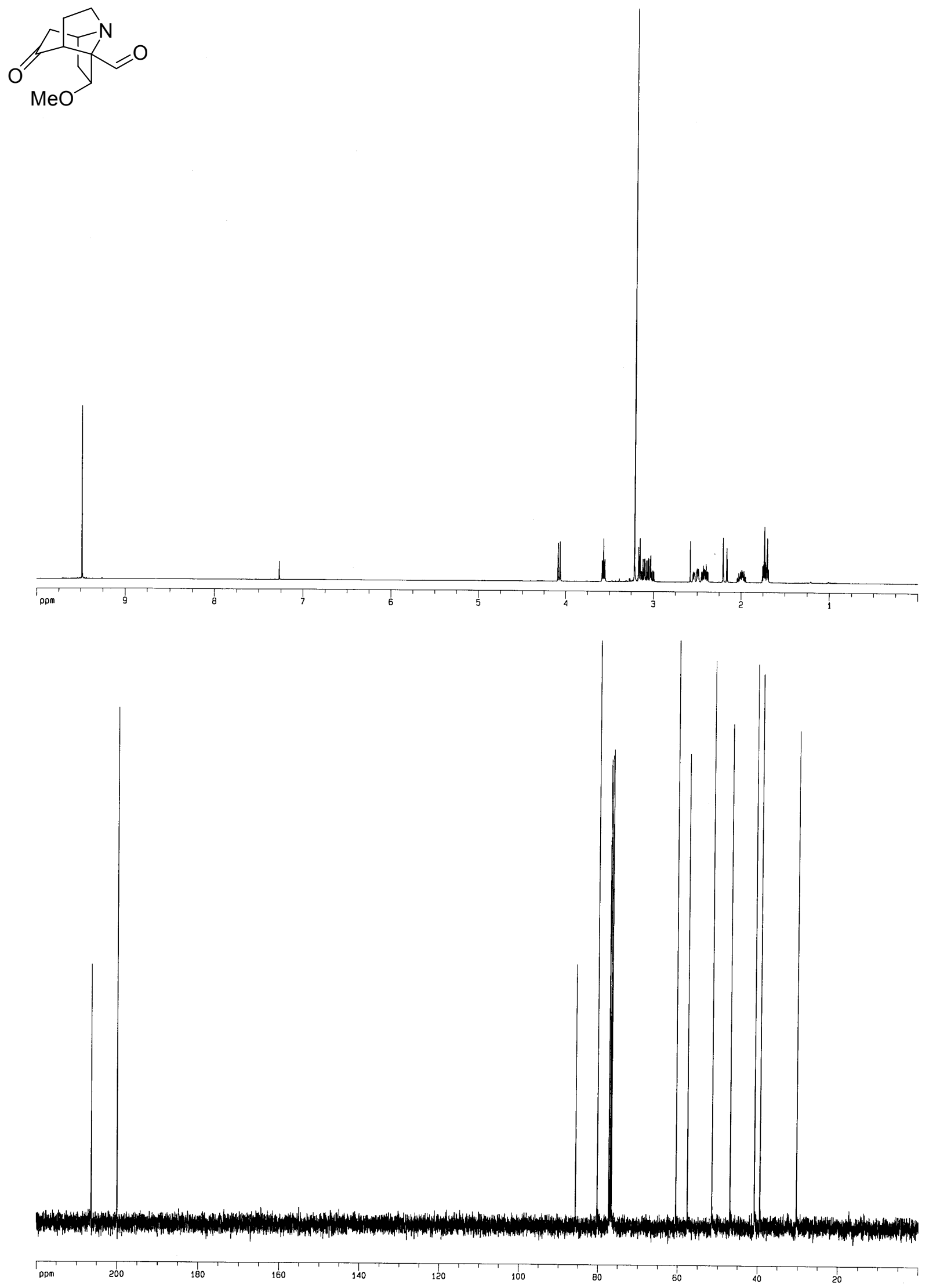
S18
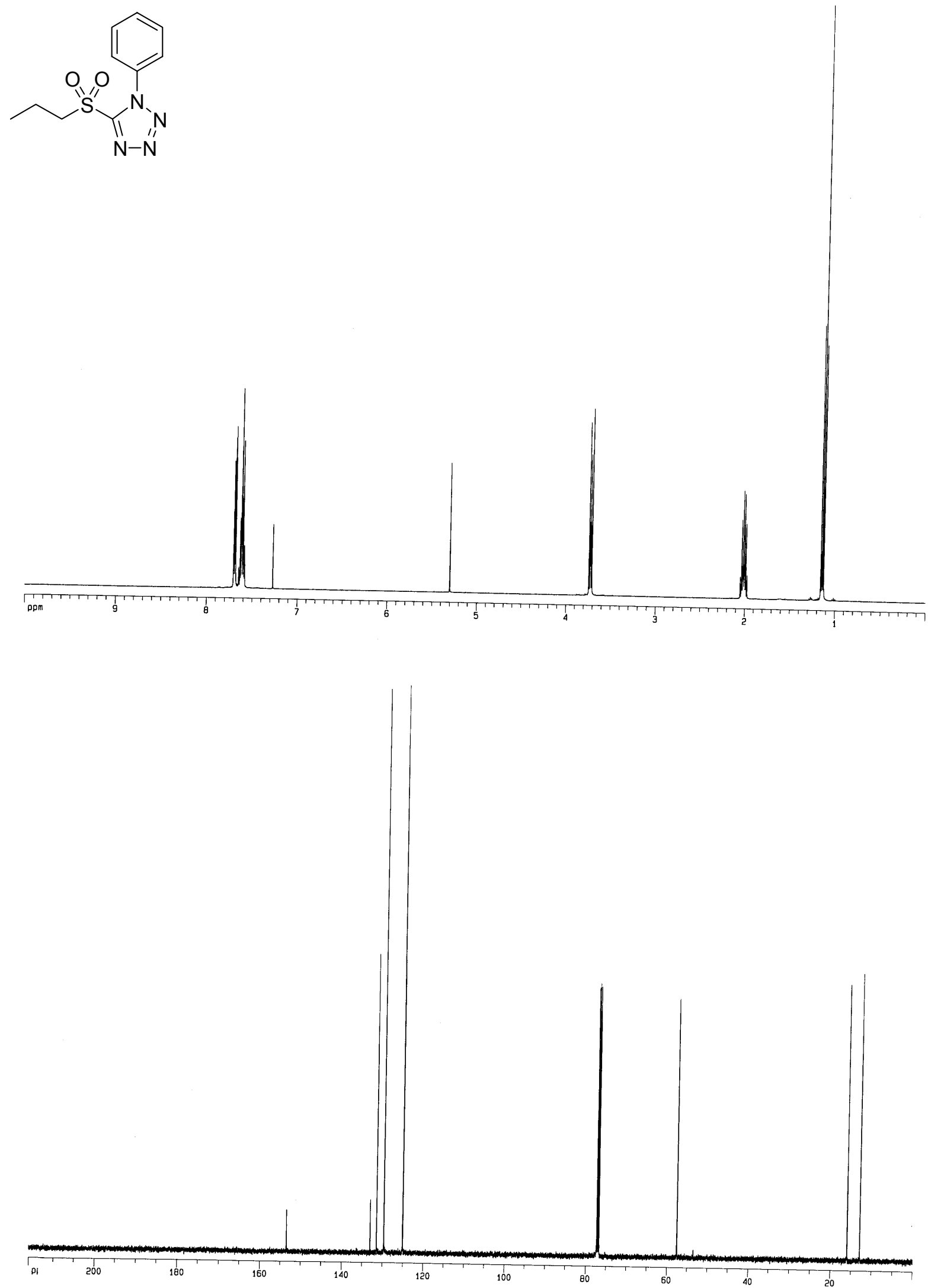
S19
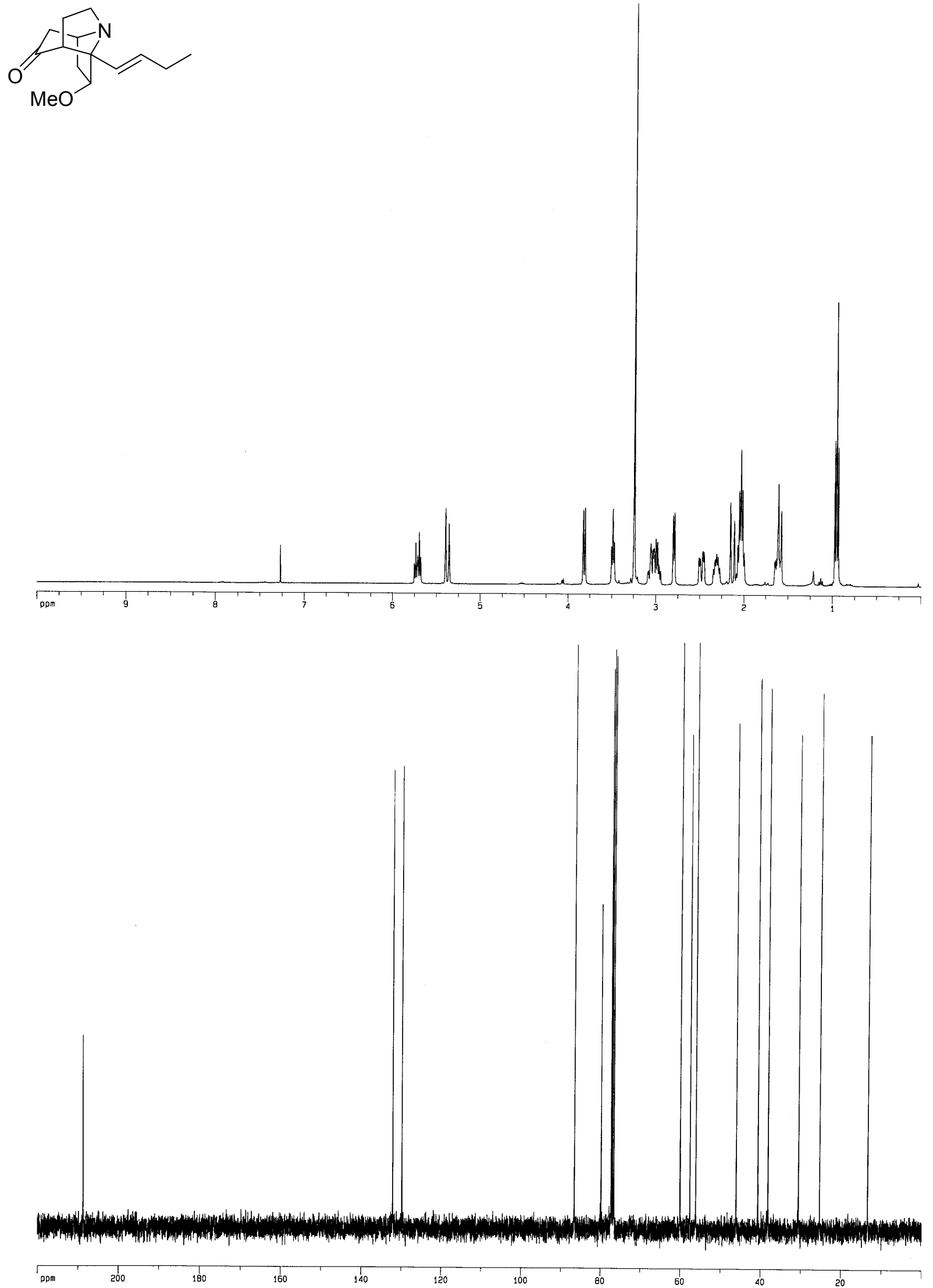
S20<smiles>CC/C=C/C12CCN(CC1CC(=O)OCC)C(OC)C2C(=O)OCC</smiles>
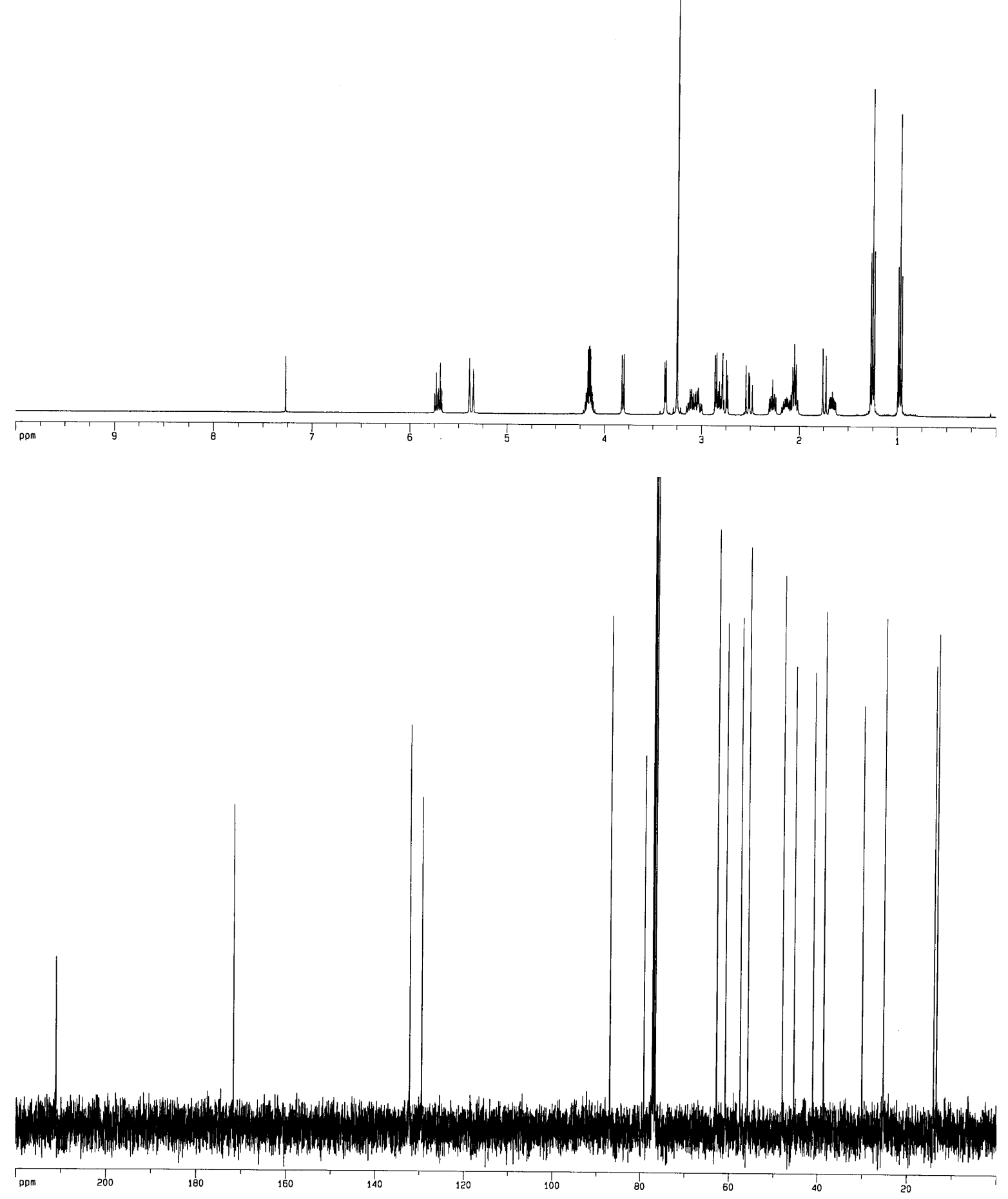
S21

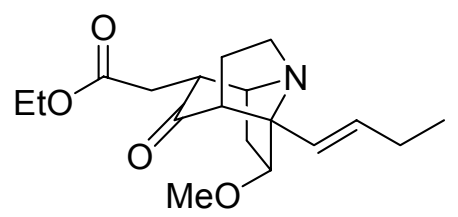
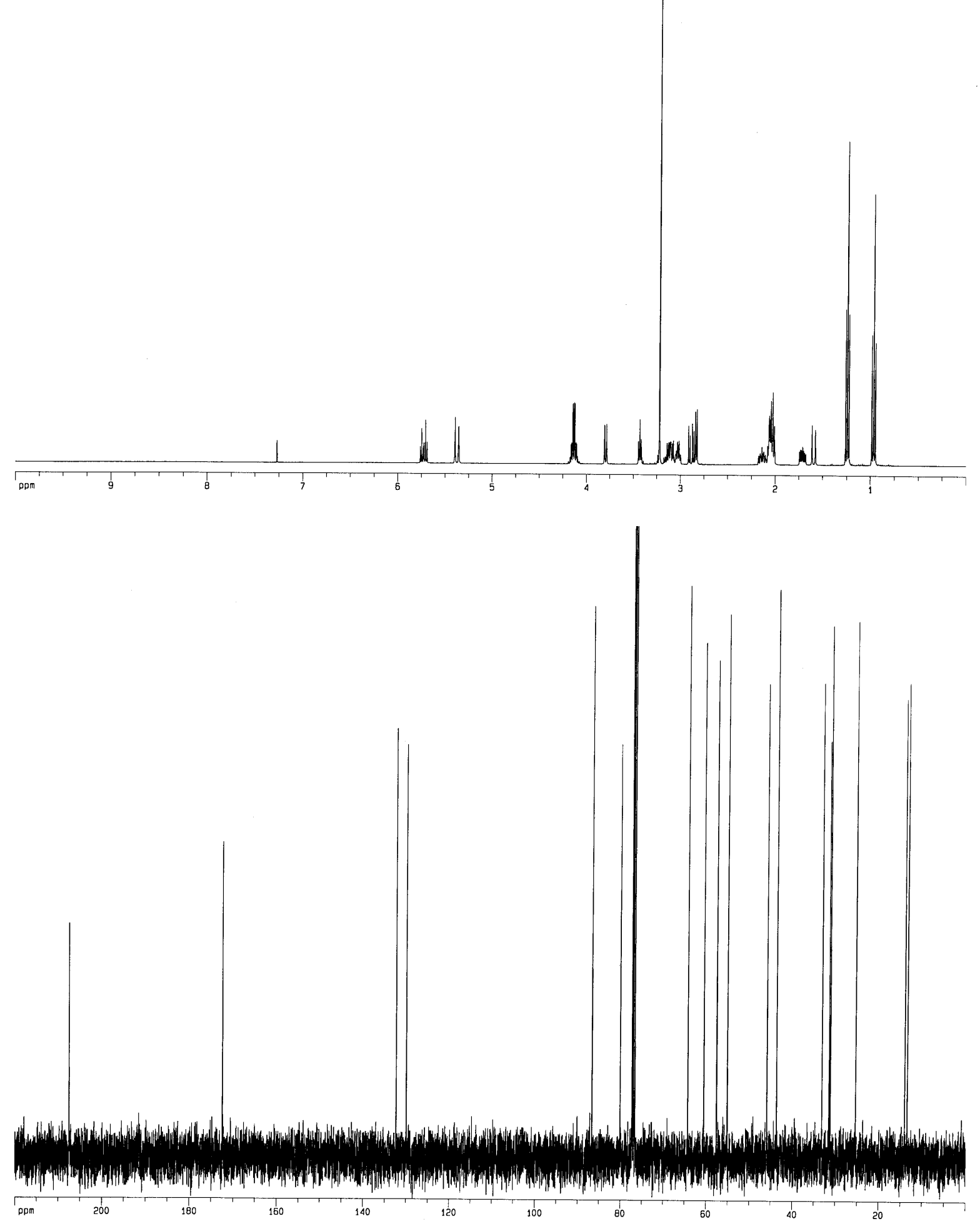
S22<smiles>CCC=CC12C3CC(CC(=O)OCC)C1OC(O)(O3)C1CCN12</smiles>
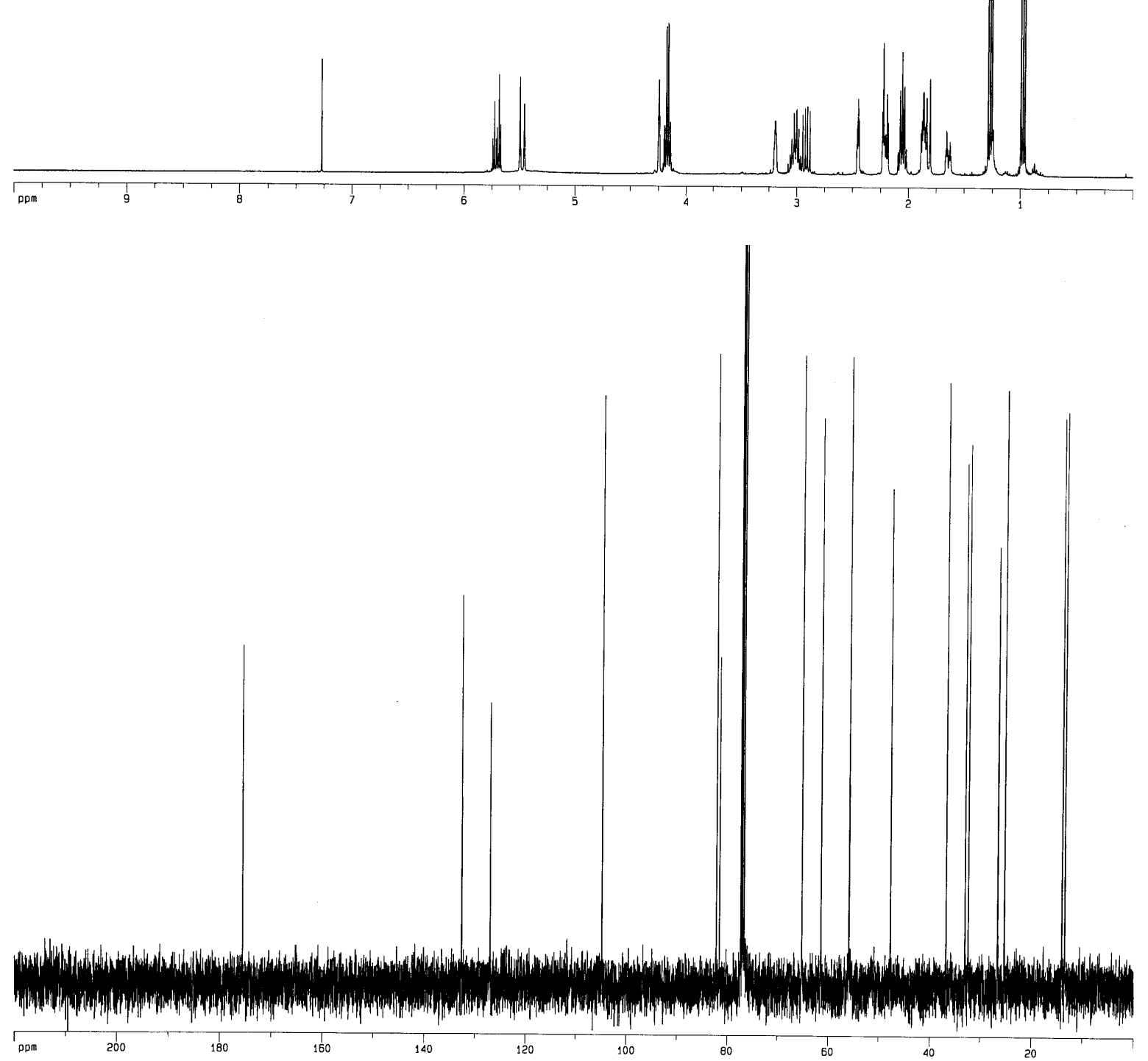


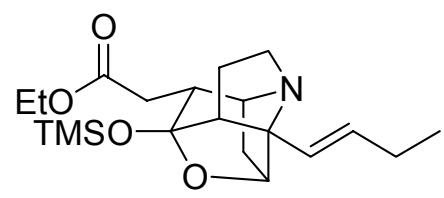
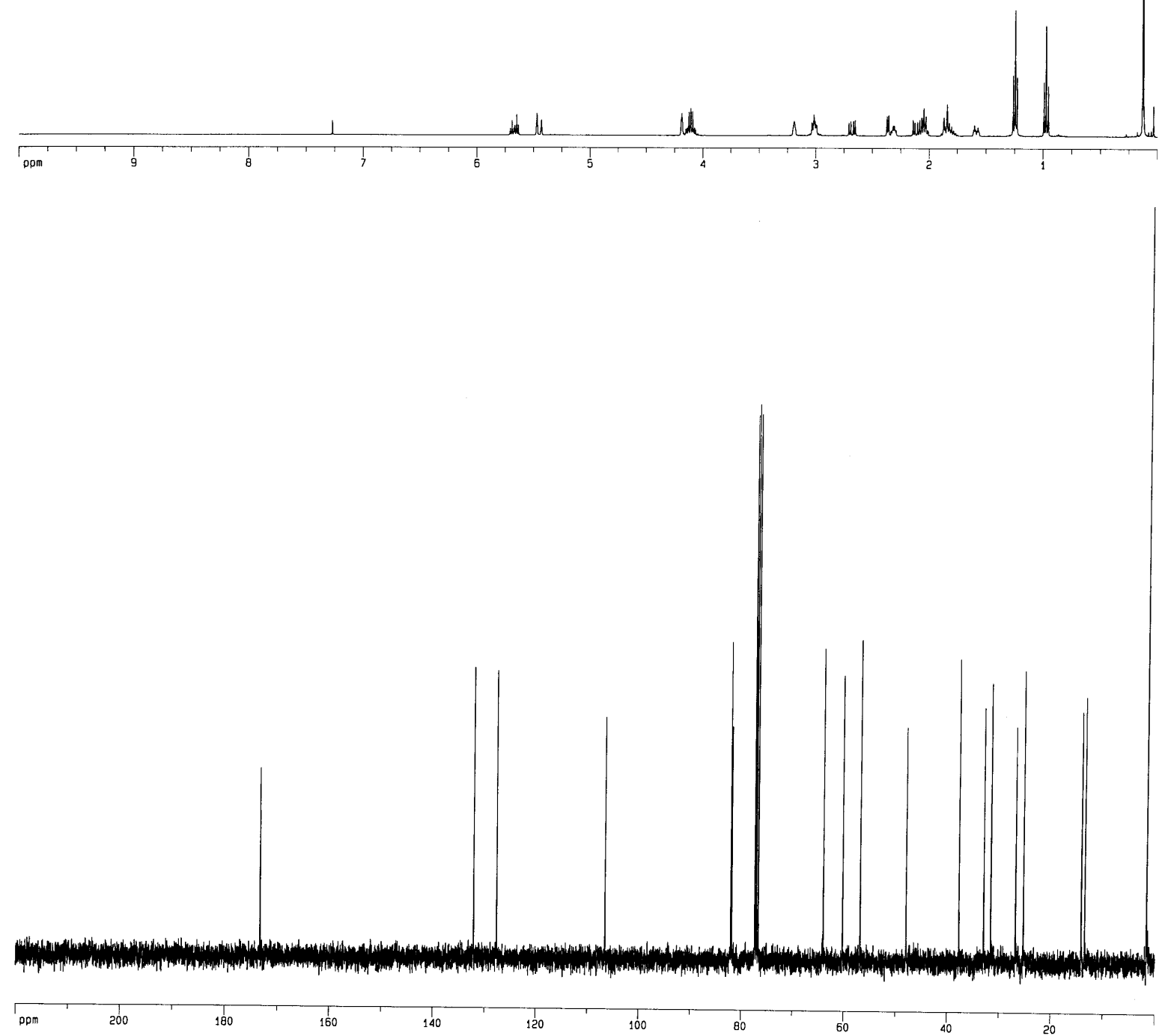

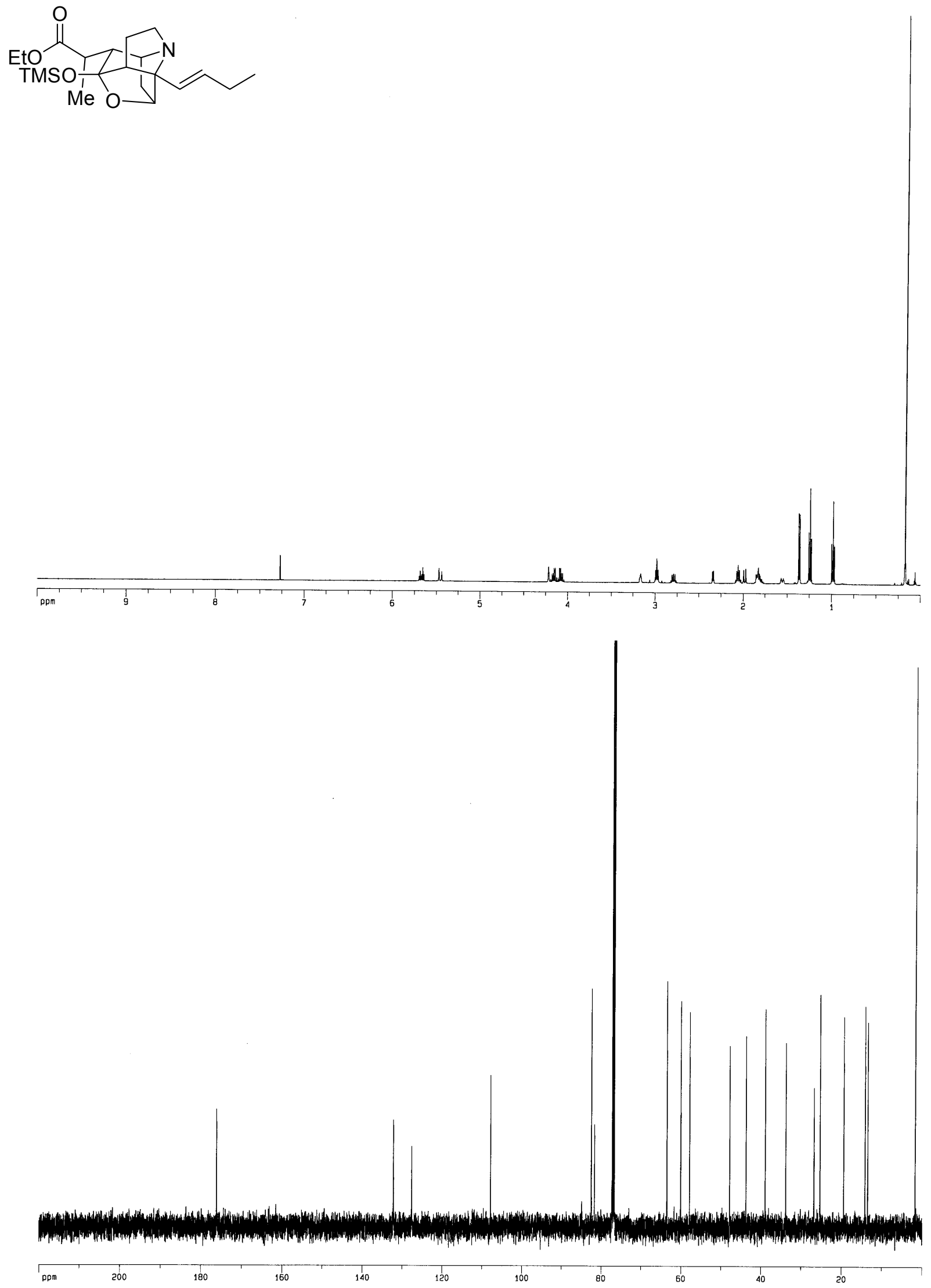

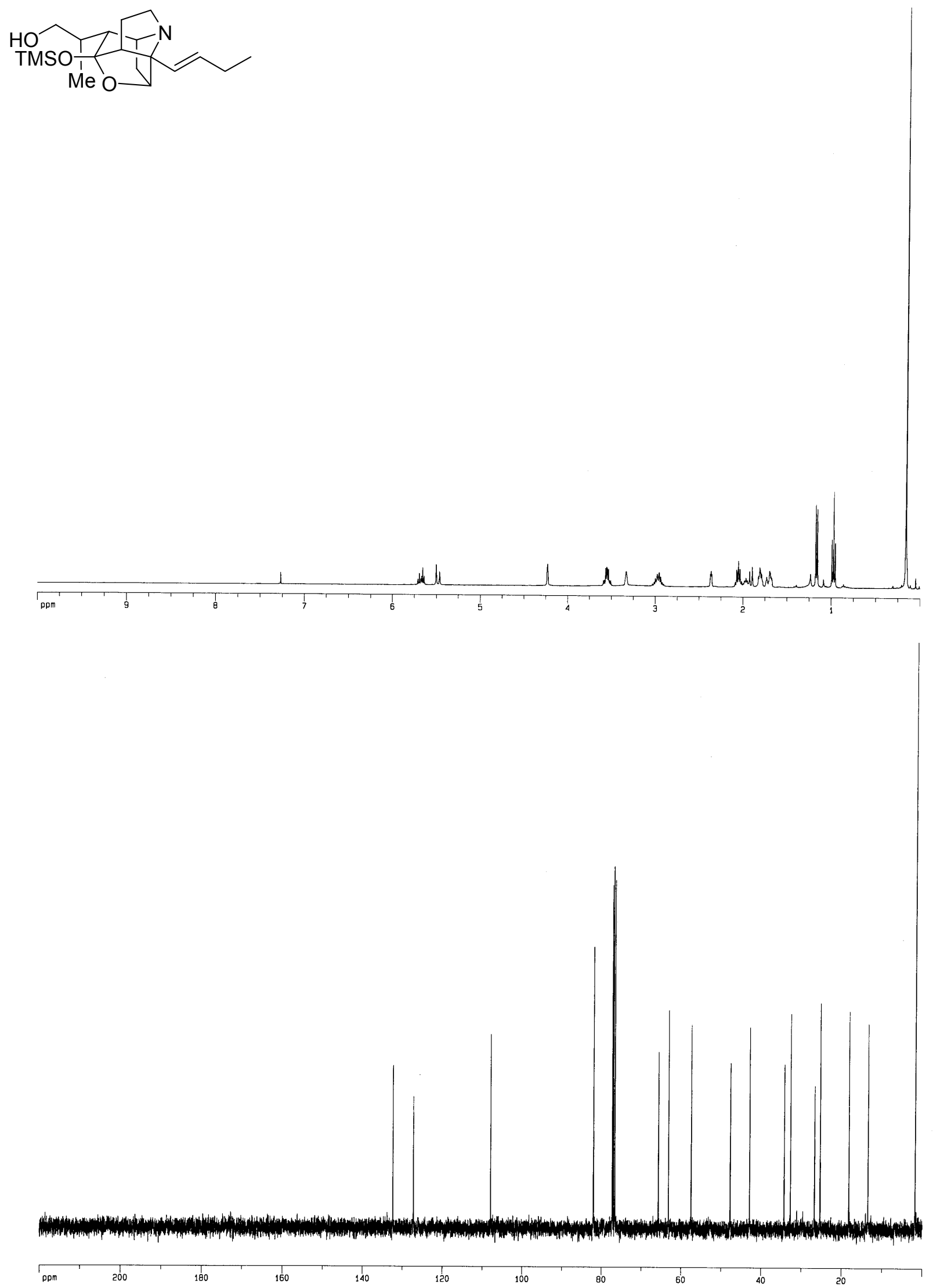
S26
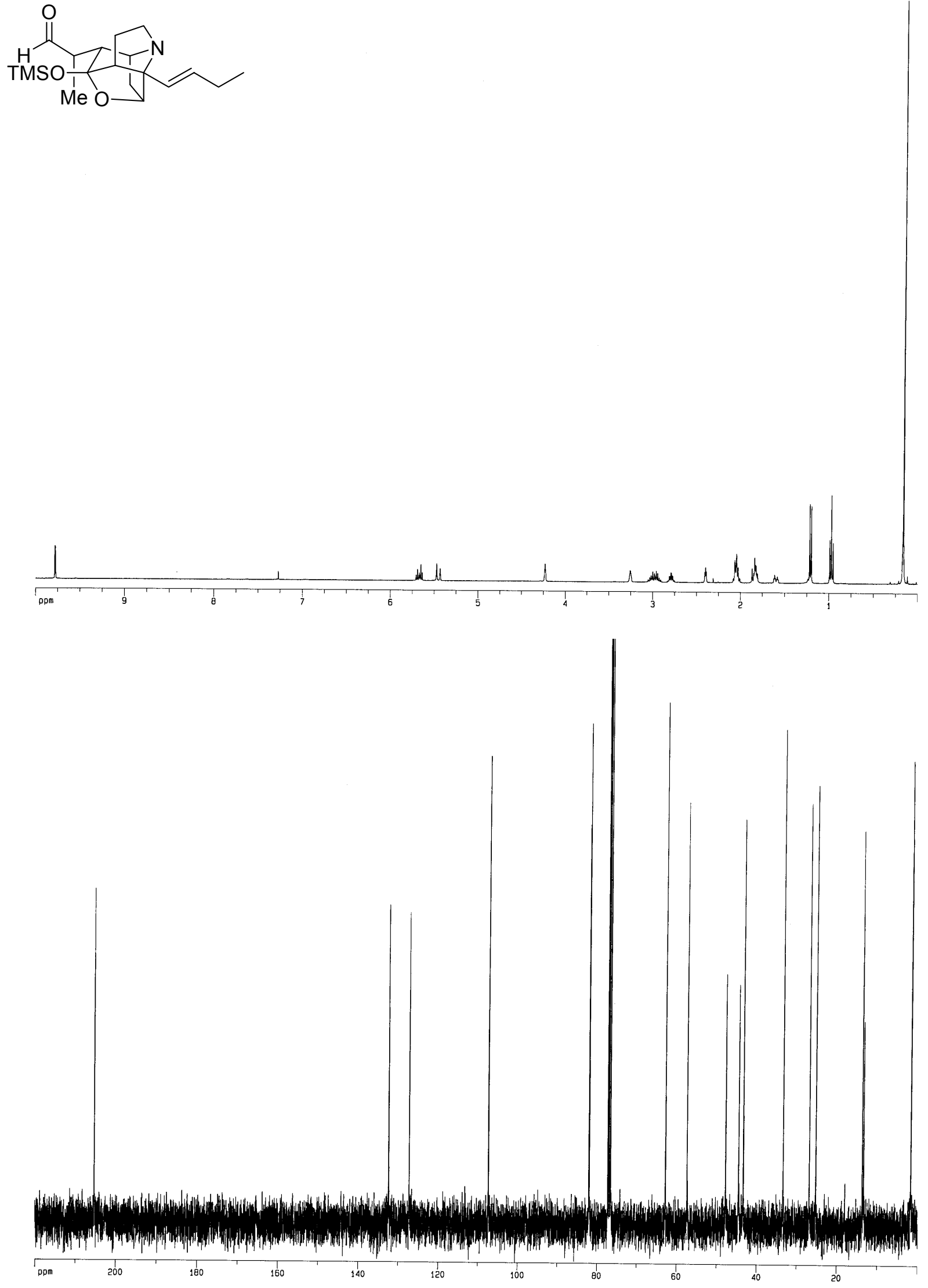

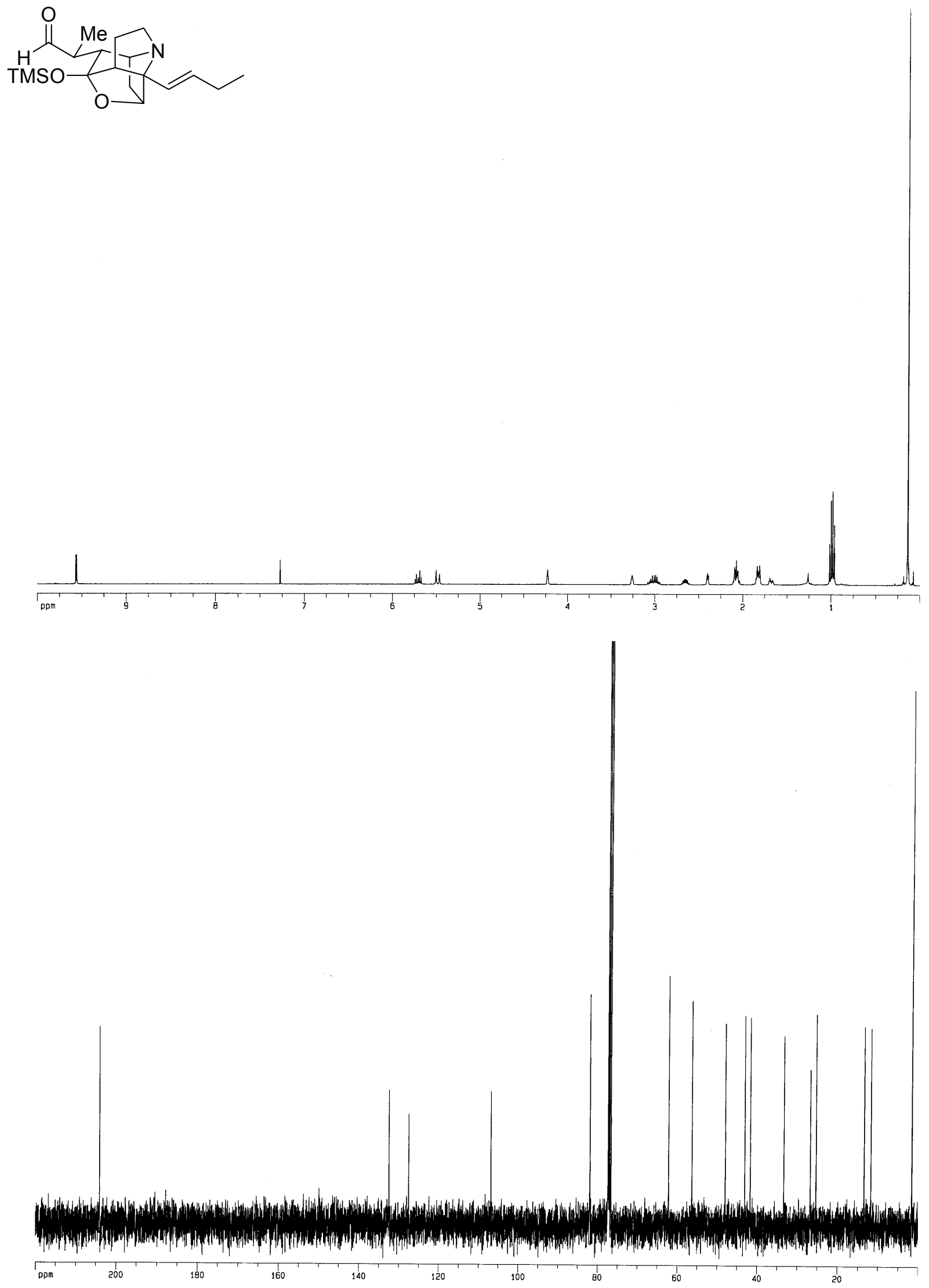

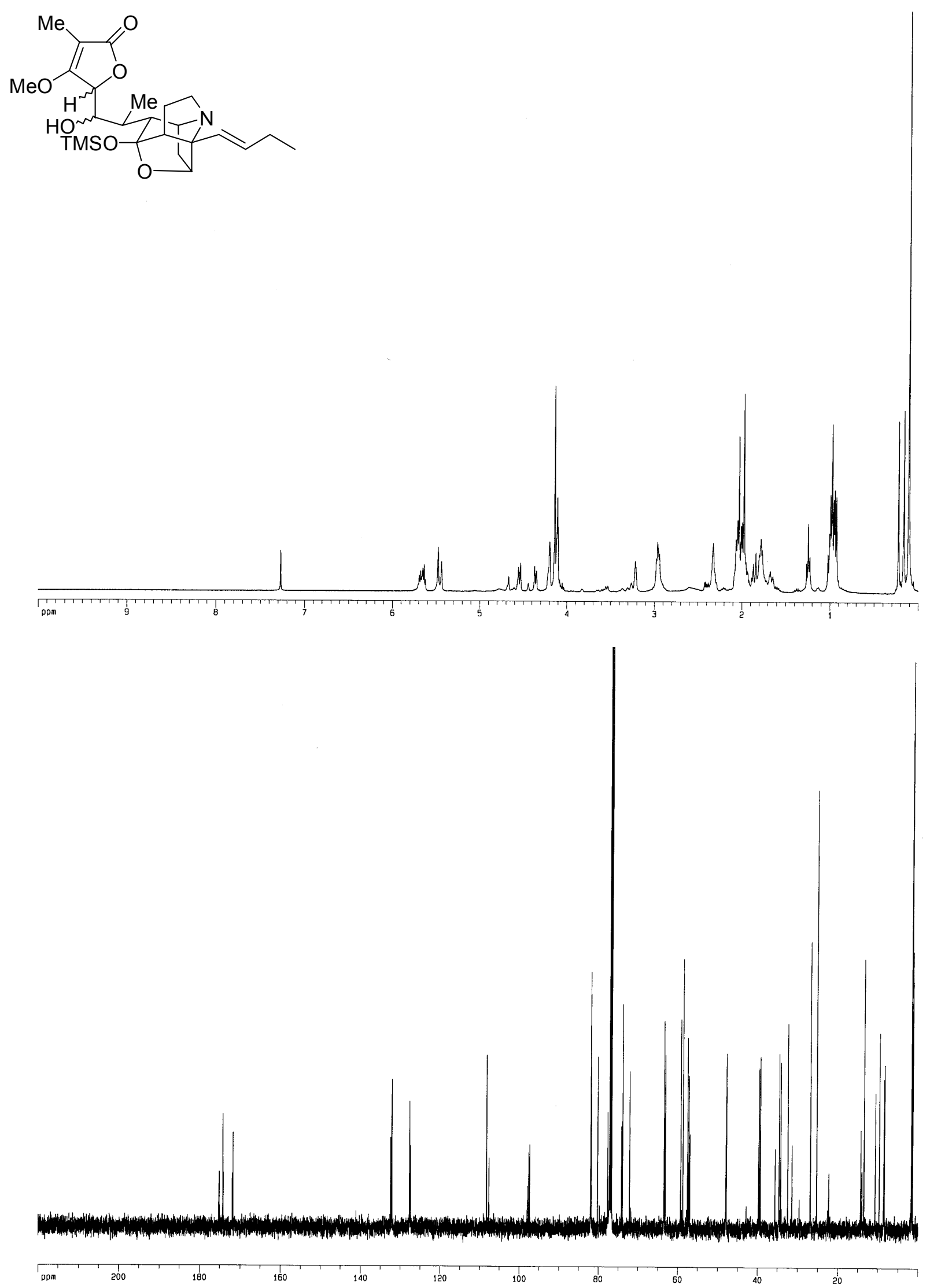
S29
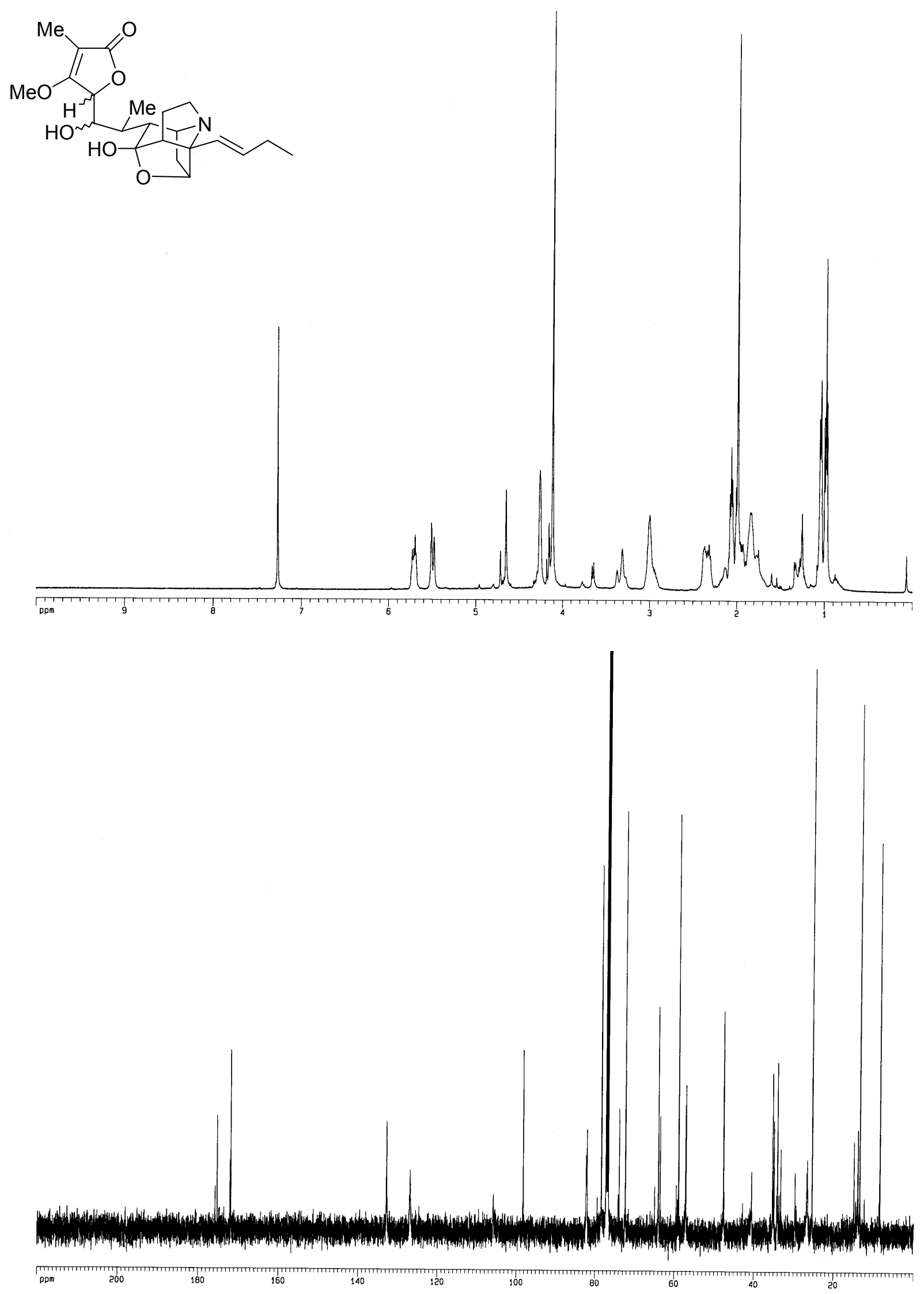
S30
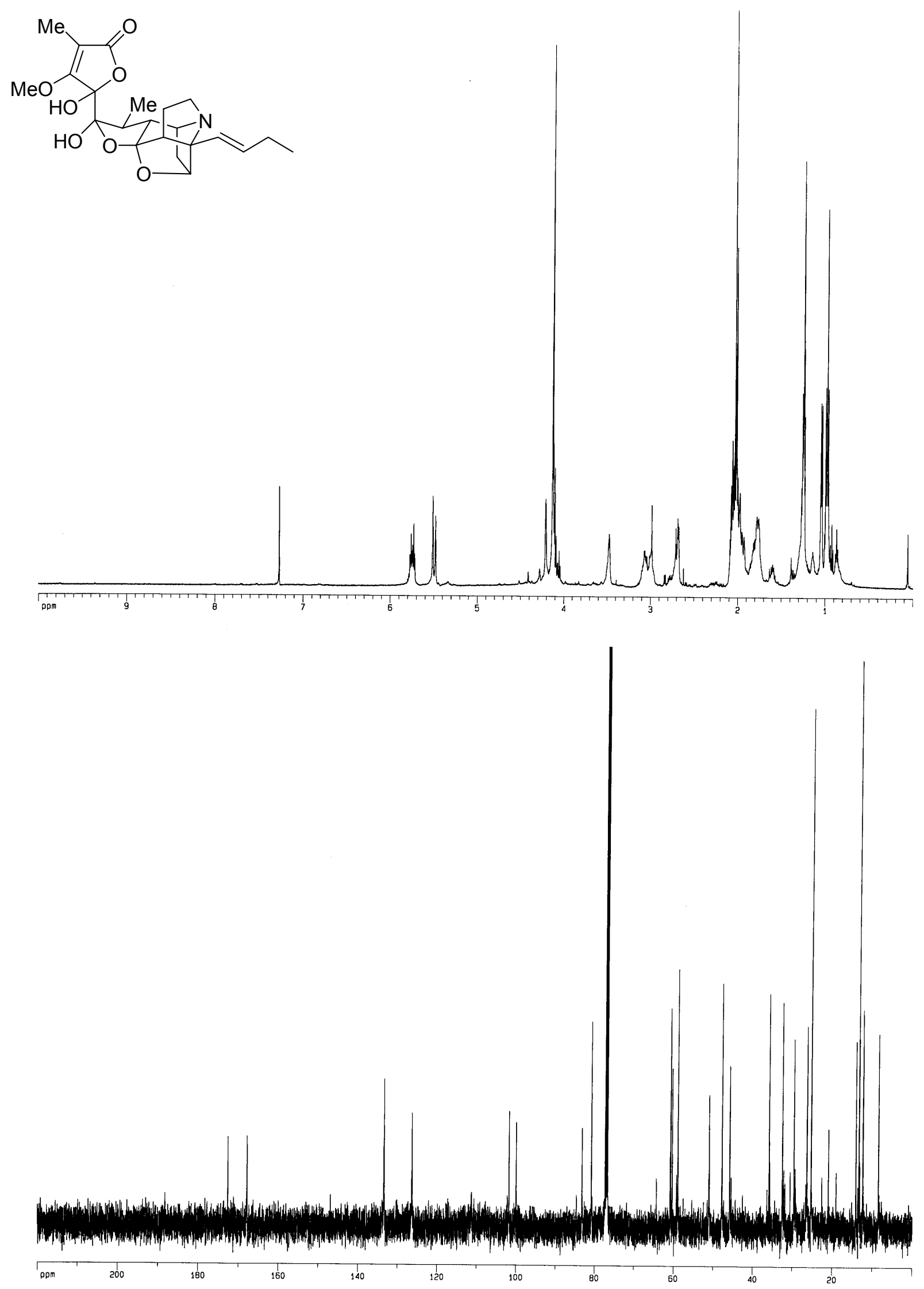

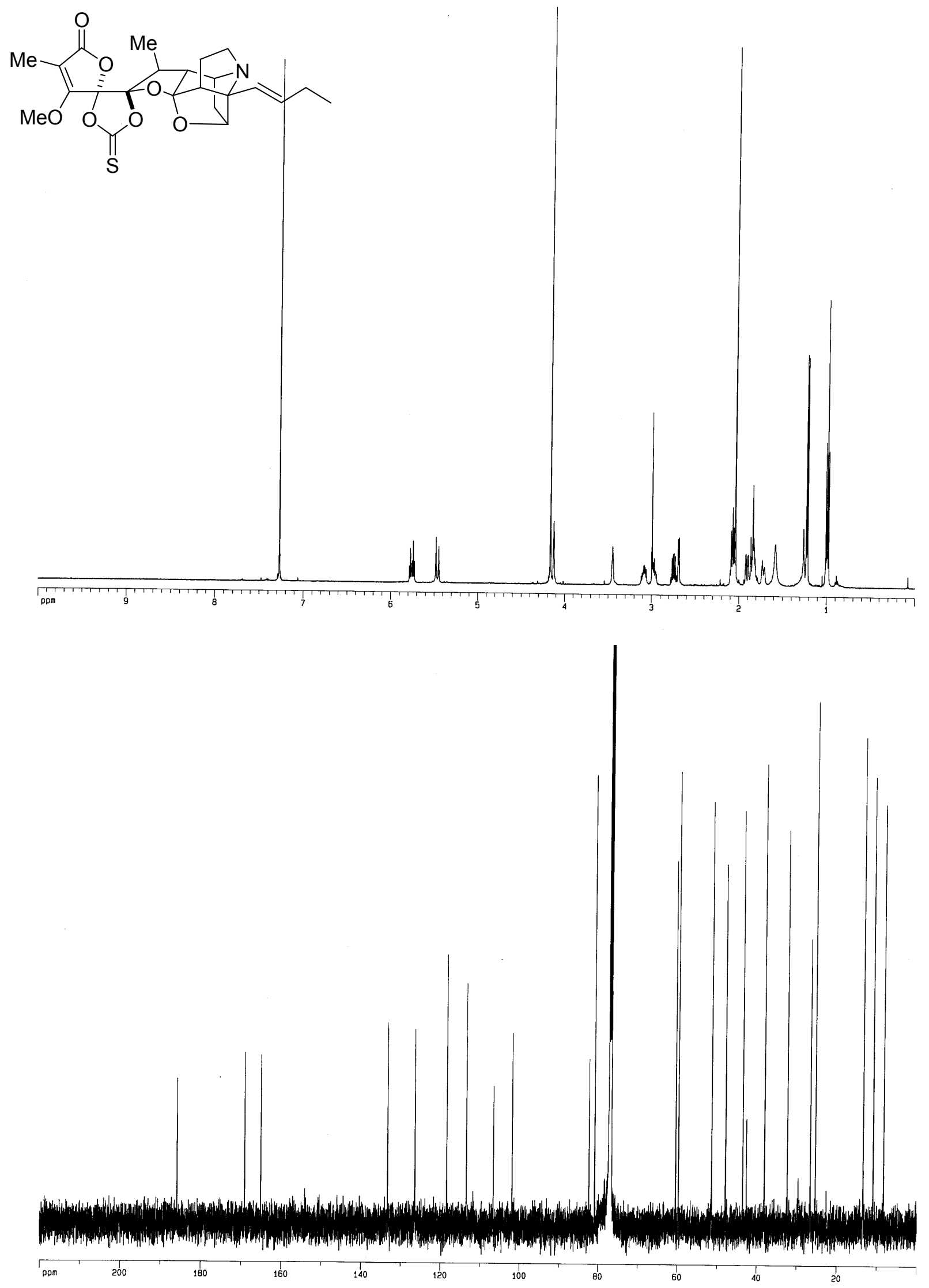

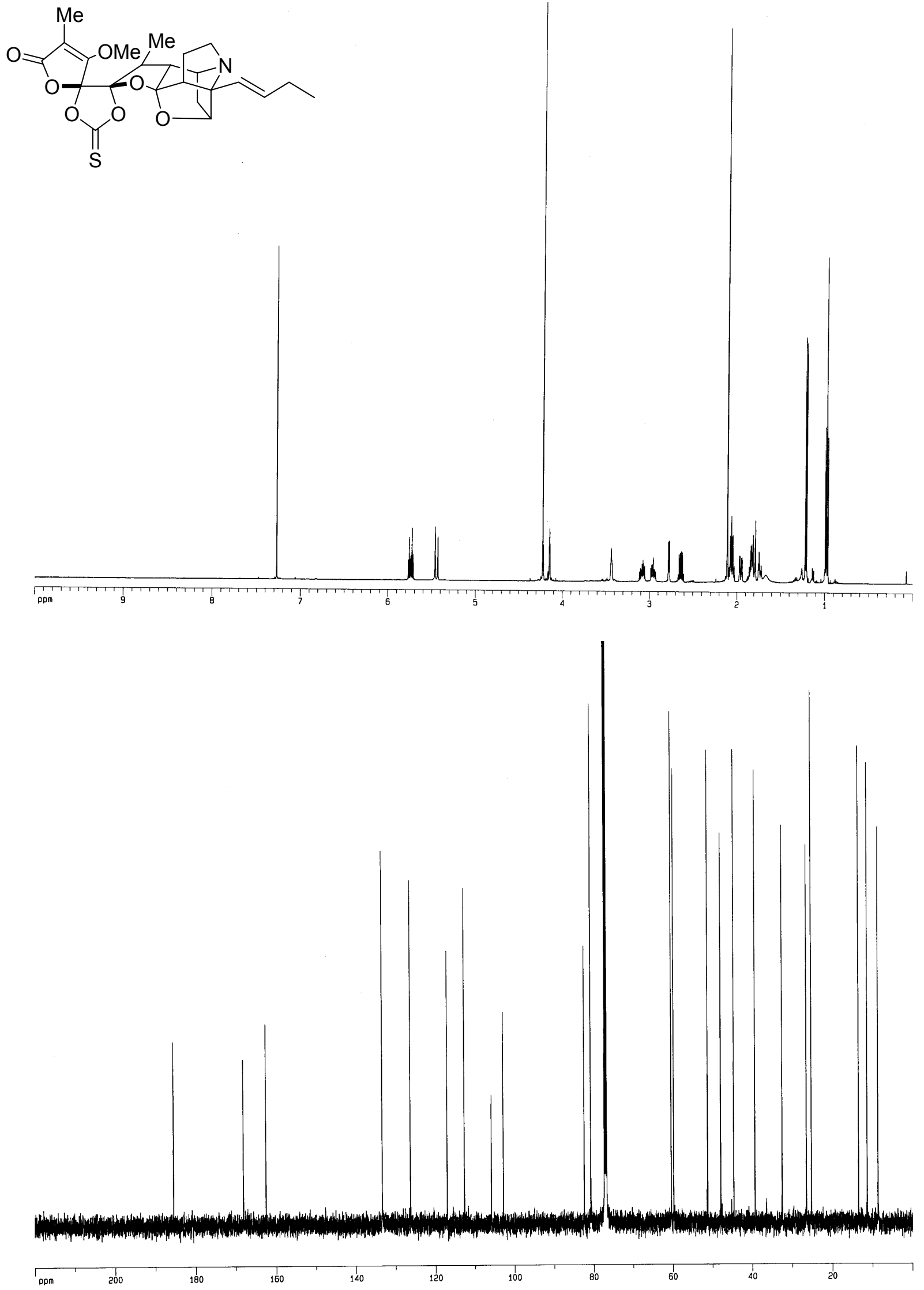


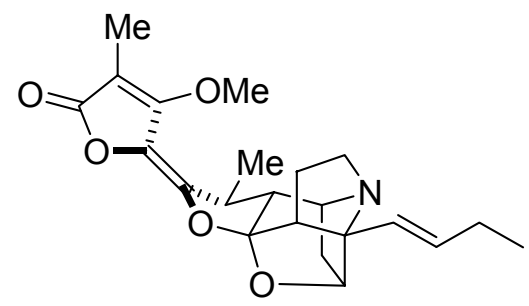

synthetic ( \pm )-didehydrostemofoline
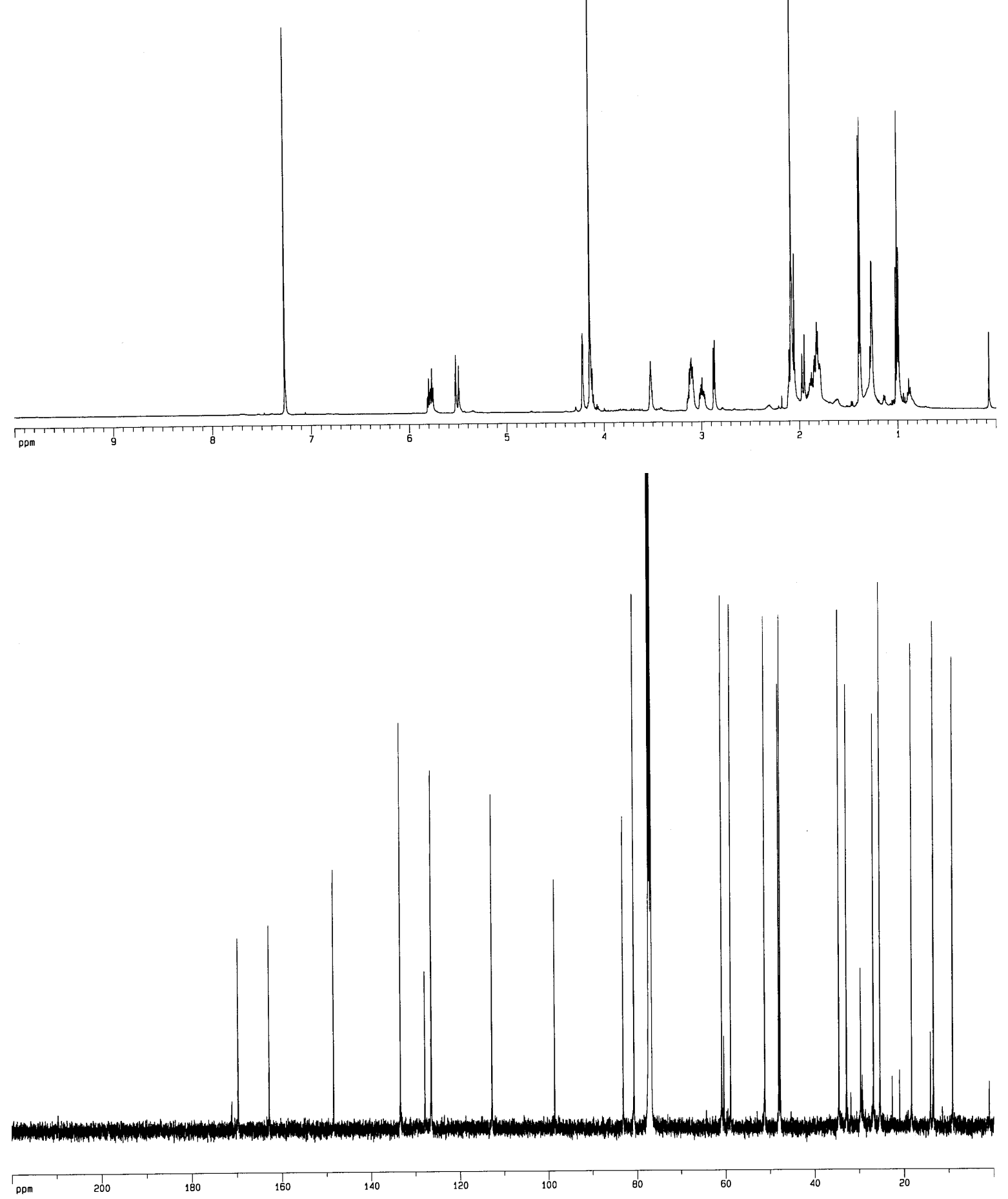


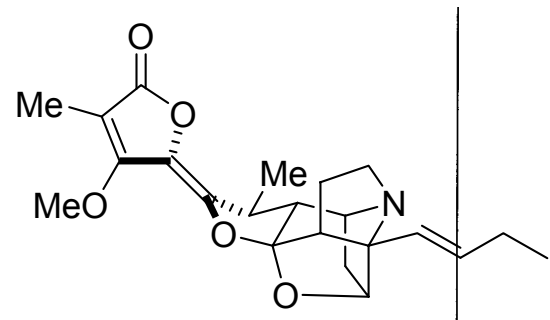

synthetic ( \pm )-isodidehydrostemofoline
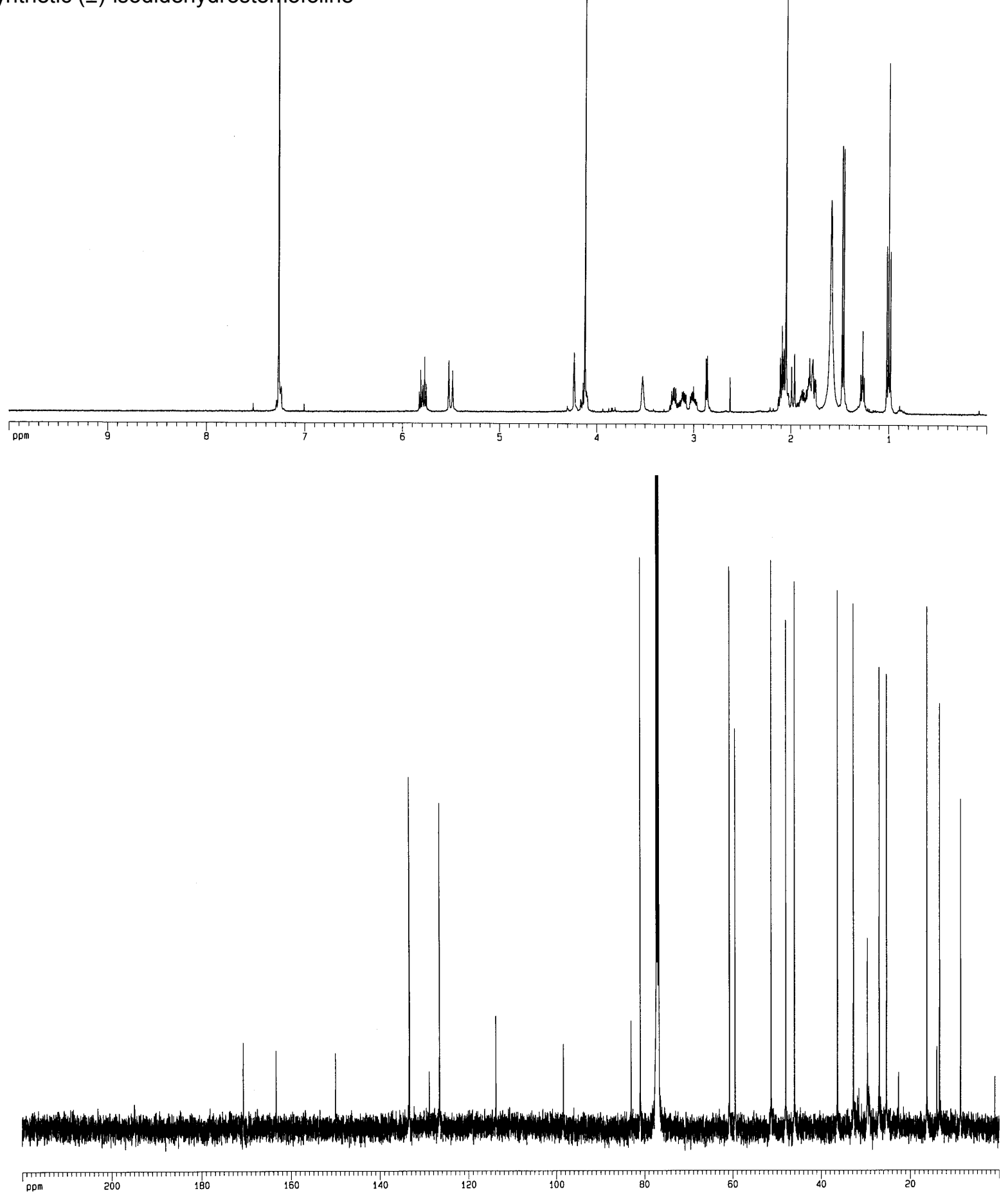